\title{
Optimal Reserve Prices in Name-Your-Own-Price Auctions with Bidding and Channel Options
}

\author{
Gangshu (George) Cai \\ Department of Management, Kansas State University, Manhattan, Kansas 66506, USA, gcai@ksu.edu \\ Xiuli Chao \\ Department of Industrial and Operations Engineering, University of Michigan, Ann Arbor, Michigan 48109, USA \\ School of Economics and Management, Tsinghua University, Beijing, 100084, China, xchao@umich.edu
}

\author{
Jianbin Li \\ School of Management, Huazhong University of Science and Technology, Wuhan, Hubei Province, 430074, China, jimlee@amss.ac.cn
}

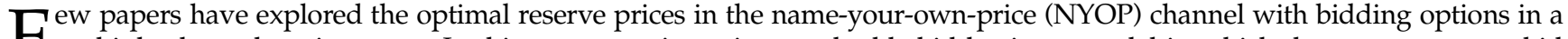
$\mathrm{F}$ multiple channel environment. In this paper, we investigate a double-bid business model in which the consumers can bid twice in the NYOP channel, and compare it with the single-bid case. We also study the impact of adding a retailer-own list-price channel on the optimal reserve prices. This paper focuses on achieving some basic understanding on the potential gain of adding a second bid option to a single-bid system and on the potential benefits of adding a list-price channel by the NYOP retailer. We show that a double-bid scenario can outperform a single-bid scenario in both single-channel and dual-channel situations. The optimal reserve price in the double-bid scenario is no less than that in the single-bid case. Furthermore, the addition of a retailerown list-price channel could push up the reserve prices in both single-bid and double-bid scenarios.
\end{abstract}

Key words: reserve price; name-your-own-price (NYOP); single-bid; double-bid; dual-channel

History: Received: November 2007; Accepted: November 2008 by Michael Pinedo; after 3 revisions.

\section{Introduction}

The name-your-own-price (NYOP) auction has become popular since the inception of Priceline in 1998, because consumers can pay less in an NYOP channel than in list-price channels. In an NYOP auction, a consumer submits a bid on Priceline, and Priceline informs the consumer whether he/she wins the bid after a short period of time. Typical items for sale on Priceline include hotel rooms, rental cars, airline tickets, and cruises.

Implied by its name and as first claimed by Priceline, NYOP sounds like a reverse auction in which sellers compete for the bid. However, "Priceline isn't an auction" (Segan 2005). Like other list-price retailers, Priceline has a given (minimum) price for every item. This price is called reserve price, and a customer wins the item only if his/her bid is higher than the reserve price (Terwiesch et al. 2005). Since consumers do not have complete information of the products/ prices, Priceline's deals are opaque. For example, the consumers cannot know the exact hotel when they bid for hotel rooms in a specified area, nor do they know whether the bidding prices will be accepted. Opaque fares are generally lower than most list-prices on the Internet, because the contracts between Priceline and its suppliers "give Priceline really low rates" (Segan
2005). However, consumers might also incur other costs, such as time, emotion, etc., which is referred to as frictional or haggling cost (Hann and Terwiesch 2003, Terwiesch et al. 2005). Thus, the consumers have to trade off their convenience with the low price. In theory, an NYOP channel could be better than a listprice channel (Fay 2004, Terwiesch et al. 2005). As argued by many researchers, the NYOP channel provides a niche market where consumers are sensitive to price or psychologically prefer this kind of auction (Clark 2000, Fay 2004, Segan 2005).

Priceline has made some significant changes over the years. For example, Priceline used to allow the consumers to repeatedly bid on the same item within several days after the first bid; however, this policy is no longer available from several years ago. Although consumers can continue to submit a different bid by modifying at least one bidding option, this alteration in policy has changed the consumer behavior and might affect Priceline's profit. Additionally, Priceline has launched a list-price channel allowing customers to buy items directly without bidding. These changes motivate the following questions: Is a single-bid scenario better than a double-bid scenario? What are the consumers' behaviors in single-bid and double-bid scenarios? What are the optimal reserve prices in the 
above scenarios? What is the impact of dual-channel on optimal reserve prices?

Existing research on NYOP auctions is recent and relatively limited. Hann and Terwiesch (2003) study consumer behavior in NYOP auctions. The bidders are allowed to submit bids repeatedly; however, by doing so, substantial frictional costs occur. Hann and Terwiesch (2003) show that consumers might have lower frictional costs by learning from previous bidding experiences. Terwiesch et al. (2005) provide dynamic programming models to identify the optimal bidding strategy for consumers who might submit multiple bids, but incur more haggling costs if they continue to bid after losing their initial bids. Terwiesch et al. (2005) obtain an optimal number of bids and the corresponding values for consumers and further suggest an optimal reserve price. The optimal reserve price is constant, which is supported by collected data and is also adopted in our model. Terwiesch et al. (2005) show that a haggling model may be better than a list-price model if the consumers are rather heterogeneous.

Fay (2004) studies a partial double-bid scenario in a slightly different NYOP. In the model, the seller announces a higher reserve price and then a lower reserve price to the consumers in sequential selling; however, the number of items for sale is determined by nature. The consumers can submit the second bid if they lose the first sale. Fay (2004) suggests that a partial double-bid could be better than a single-bid for the retailer although the result is conditional on some restriction. In his model, a list-price is better than an NYOP for the retailer; however, as explained by the author, an NYOP has its advantages, e.g., the retailer might collect more information about the consumer demand and it might appeal to a "segment that receives a psychological benefit."

In a model to find the haggling cost for consumers, Spann et al. (2004) allow repeat bidding in the NYOP channel. With unlimited inventory capacity, they suggest that repeated bidding can be better than single bidding for the seller. In an experimental work, Spann et al. (2005) assume that "consumers are often uncertain about their exact valuations of a particular product." Given that only one bid is allowed, consumers can exchange the information of how much they wish to bid with other bidders in three different experimental designs: name-your-price, select-yourprice low range, and select-your-price high range. Consumers then predict the optimal bid based on the price elicitation. Spann et al. (2005) suggest that the form of price elicitation has a significant impact on the seller's profit. Chernev (2003) also explores the price elicitation in reserve pricing by showing that select-your-price might be better than name-yourprice in several experiments because, as he explains from a psychologic perspective, the name-your-price approach "is likely to be associated with a greater degree of uncertainty and cognitive effort." Based on the data of NYOP for airline tickets, Spann and Tellis (2006) classify the consumers' bidding into different patterns. In a model that the consumers can revise their bids based on the winning probability function provided by the retailer, Wilson and Zhang (2008) show that there exists an $\varepsilon$-optimal solution for the retailer to design the winning probability function.

While the literature has been focused on a single NYOP channel, few have studied the coexistence of an NYOP channel and list-price channels. Ding et al. (2005) study a Priceline-like reverse auction by assuming that consumers might buy from list-price market, if they fail to win in the NYOP channel. Ding et al. (2005) suggest that bidders are emotional because they will feel excited when winning and frustrated when losing. Thus, a consumer incurs an emotional utility, in addition to a simplified monetary utility, that is linear to the difference between the bid and the listprice. Wang et al. (2005) assume that a bidder's valuation is discounted due to the opacity of the NYOP channel, which allows a single-bid. It is the seller's task to optimize the opacity in the NYOP channel. Note that the opacity is identical for every customer once the seller determines the optimal level. The service provider in their model is a monopolist, such that all unsatisfied bidders buy from his/her own direct listprice market. However, due to their special focuses, Ding et al. (2005) and Wang et al. (2005) do not explicitly consider the double-bid scenario. Other work on multi-channel supply chains (Cai et al. 2009, Caldentey and Vulcano 2007, Chen et al. 2007, Chiang et al. 2003, Etzion et al. 2006, Tsay and Agrawal 2004, van Ryzin and Vulcano 2004, Zhao 2008) and auctions (Cai and Wurman 2005, Chen et al. 2008, Rothkopf and Whinston 2007, Shen and Su 2007) does not consider NYOP auctions.

In this paper we study the optimal reserve prices in a variety of situations, including different combinations of single-channel, dual-channel, single-bid, double-bid, abundant inventory capacity, and constrained inventory capacity in the NYOP channel. We show that a double-bid scenario can outperform a single-bid scenario in both single-channel and dual-channel situations. The optimal reserve price in the double-bid scenario is no less than that in the single-bid case. The addition of a retailer-own list-price channel could push up the reserve prices in both single-bid and double-bid scenarios. We obtain the conditions where the doublebid is a better choice for the retailer than the single-bid. We further suggest that the double-bid may become more significant if it can attract more consumers in a dual-channel environment.

The remainder of this paper is organized as follows. In Section 2, we present the model. In Section 3, we 
describe the single-bid scenario. We study the doublebid scenario and compare it with the single-bid scenario in Section 4. Research conclusions are presented in Section 5, and all proofs are relegated to the Appendix.

\section{The Model}

The following notation is used in the subsequent analysis:

$Q_{0} \quad$ capacity quote for the NYOP auctions by either the supplier or the retailer

$x \quad$ a random consumer's bid, including the premium charged by the retailer

B list-price from either the retailer or competitors

$\theta \quad$ consumer preference of shopping directly from the list-price channel

$[c, d]$ domain of $\theta$

$[a, B]$ domain of a consumer's belief in winning an NYOP auction by bidding $x$

C total cost of a customer when purchasing an item

$R \quad$ reserve price that the retailer sets for a specific item in the NYOP channel

$w \quad$ wholesale price to the retailer from a specific supplier

$C_{f} \quad$ fixed cost of adding a list-price channel to the existing NYOP channel

$\lambda$ ratio of customers who fail in NYOP buy from the NYOP-retailer-own list-price channel

We consider both single-channel and dual-channel market configurations, as illustrated in Figure 1. In the first market configuration, the retailer only offers NYOP auctions to the consumers, who will buy from other competitors' list-price channel(s) if they fail to win in the auctions. In the second market configuration, the retailer provides an NYOP channel along with a list-price channel. Among these consumers who do not win from the NYOP channel, we assume

Figure 1 Market Configurations of the Retailer's Single-Channel and DualChannel Scenarios

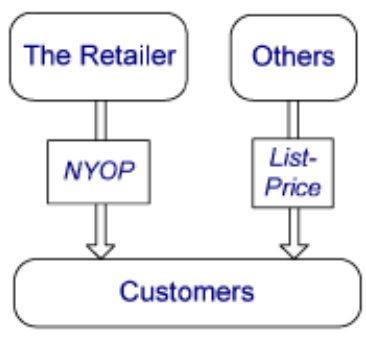

Single-Channel Scenario

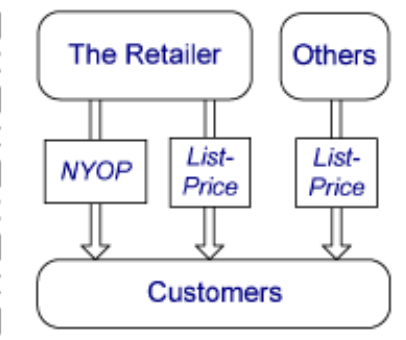

Dual-Channel Scenario that a percentage, $\lambda, 0 \leq \lambda \leq 1$, of them buy directly from the NYOP-retailer-own list-price channel while the remaining $1-\lambda$ of them buy from other competitors. Both market configurations have been observed in Priceline.com history. In line with Fay (2004) and without loss of generality, the total number of consumers, regardless of whether they win in NYOP auctions or not, is normalized to 1 .

Suppose the consumer has a winning probability of $F(x)$, called belief probability, if the consumer bids $x$. The support of $F(x)$ is on the interval $[a, B]$. This winning probability is similar to the distribution function for a threshold price in Fay (2004), Hann and Terwiesch (2003), Terwiesch et al. (2005), and has been seen in practice by some NYOP retailers, such as Priceline.com (Allbusiness.com 1999). Let $f(x)$ be the corresponding probability density function (PDF).

The retailer and the supplier have agreed on a fixed wholesale price, as reported by Segan (2005). Let $w$ denote the wholesale price, and to simplify the discussion, we assume that $w$ has absorbed other variable costs to the retailer. We normalize the fixed cost of the NYOP channel to 0 , but let $C_{f}$ denote the fixed cost to the retailer for adding the list-price channel to the existing NYOP channel.

Clearly, the consumer has no information about the reserve price $R$, which is set by the retailer in advance (Hann and Terwiesch 2003). In other words, the consumer's belief of the winning probability is independent of the actual reserve price $R$. For the retailer, if $x<R$, the bid is rejected; otherwise, the bid is accepted. We assume that $R$ remains the same for a specific item during the auction. This assumption is consistent with realistic data, see Hann and Terwiesch (2003), Spann et al. (2004), and Terwiesch et al. (2005). To avoid triviality, we assume that $\max \{a, w\} \leq$ $R \leq B$.

The NYOP auctions have opaque prices. According to Segan (2005), the retailer treats its customers as price-sensitive travelers who are willing to give up some convenience for a lower price. For example, consumers do not know exactly what hotel or location for which they are bidding. Different consumers have heterogeneous preferences toward these opaque fares. Let $\Theta$ be the preference of an arbitrary consumer toward the list-price channel, which is a random variable. When $\Theta=\theta$, then $\theta$ is the disutility incurred by the consumer for losing the convenience when winning the item from the NYOP. Assume that the consumer preference $\Theta$ is uniformly distributed on an interval $[c, d]$. Typically $c \geq 0$, which means that consumers prefer the list-price to the NYOP in general, if the price is the same in both channels. The listprice is denoted by $B$ and is assumed to be the same in both the NYOP-retailer-own list-price channel and the competitors' list-price channels, which can be 
observed on Priceline and other list-price competitors, e.g., Hotels.com.

We investigate two scenarios. The first one is called single-bid scenario, in which all consumers can bid only once in the NYOP. The second is called double-bid scenario, in which all consumers can bid twice in the NYOP. Current practice on Priceline is widely considered as a single-bid scenario, while a scenario of multiple (more than two) bids was abandoned by Priceline several years ago. However, it remains arguable whether the retailer should allow one more bid in the NYOP auction since the consumers could still bid a second time by using a different user name, changing the credit card number, and so on. Similar to Fay (2004), we compare these two scenarios but in a different context.

In addition to the study of the abundant capacity case, we consider the impact of an inventory capacity quote $Q_{0}$ on the NYOP channel. This capacity quote may be enforced by either the supplier, due to the low price caused by the opacity, or by the retailer to maximize the profit. Note that $Q_{0}$ is the normalized capacity, not the real capacity, since the number of customers has been normalized to 1 .

\section{A Single-Bid Scenario}

In line with other work (Caldentey and Vulcano 2007, Etzion et al. 2006) on multi-channel marketing, we assume that a particular consumer first bids in the NYOP auction and then buys directly from the listprice channel if he/she fails in the NYOP auction. Since the consumer has only one chance to win in the NYOP auction, to minimize the total expected cost (Wilson and Zhang 2008), we have

$$
\begin{aligned}
& \min _{x} C(\theta)=F(x)(x+\theta)+(1-F(x)) B \\
& \text { s.t. } \quad a<x<B .
\end{aligned}
$$

Substituting $F$ by the uniform distribution and optimizing the above equation without considering the constraint yields

$$
x^{*}=\frac{B-\theta+a}{2} .
$$

Clearly, the optimal bid increases in $B$ and $a$ but decreases in $\theta$. The existence of $x^{*}$ requires $a \leq x^{*} \leq B$ which is equivalent to $a-B \leq \theta \leq B-a$. The case of $a-B \leq \theta$ is trivial, since $a-B \leq 0 \leq c$. Consider $\theta \leq B-a$. If a consumer's preference to the list-price is larger than $B-a$, the consumer does not bid on NYOP. This assumption can be supported by the fact that people still buy flight tickets directly from major airlines, opposed to the NYOP auctions on Priceline, because they strongly prefer exact flight times and specific airlines. Excluding such consumers, we can without loss of generality assume $B-a \geq d$. That is to say, if a bidder is willing to bid $a$ at NYOP, his/her preference to the list-price channel should not be larger than $d$. We utilize this condition throughout this paper. Thus, Equation (1) holds with the constraint.

The consumer demand to the NYOP channel, $D_{N Y O P}$, can be described by comparing the consumers' optimal bids with the reserve price as follows:

$$
\begin{aligned}
D_{\text {NYOP }}=\operatorname{Pr}\left(x^{*} \geq R\right)=P(\Theta \leq B+a-2 R) \\
= \begin{cases}1, & \text { if } R \in\left[w \vee a, \frac{B+a-d}{2}\right) ; \\
\frac{B+a-2 R-c}{d-c}, & \text { if } R \in\left[\frac{B+a-d}{2} \vee w, \frac{B+a-c}{2}\right] ; \\
0, & \text { if } R \in\left(\frac{B+a-c}{2} \vee w, B\right],\end{cases}
\end{aligned}
$$

where $\vee$ is a maximization notation exemplified by $w \vee a=\max \{w, a\}$. The demand is constrained by $w$, because it is subject to $R \geq w$. We first explain the second case where $R \in\left[\frac{B+a-d}{2} \vee w, \frac{B+a-c}{2}\right]$. A consumer wins an item if his/her bid is higher than the reserve price, i.e., $x^{*}=\frac{B-\theta+a}{2} \geq R$, which is equivalent to $\theta \leq B+a-2 R$. Hence, the portion of all consumers who eventually win items is given by $\frac{B+a-2 R-c}{d-c}$. The expected number of customers who qualified as the second case can be expressed as $\frac{B+a-2 R-c}{d-c}=$ $\frac{B+a-c}{d-c}-\frac{2}{d-c} R$, which is a linear demand function with respect to $R$. In this sense, the maximal market size of NYOP is given by $\frac{B+a-c}{d-c}$ and $\frac{2}{d-c}$ is the decreasing rate in terms of the reserve price. For the first case where $R \in\left[w \vee a, \frac{B+a-d}{2}\right)$ given that $w \vee a<\frac{B+a-d}{2}$, all consumers win in NYOP auctions. In fact, if $R^{*}<\frac{B+a-d}{2}$, there will be multiple optimal reserve prices such that any value in $\left[w \vee a, \frac{B+a-d}{2}\right)$ is an optimal reserve price, because $D_{N Y O P}=1$. Given that $a<\frac{B+a-d}{2}$ conditional on $B-a \geq d$, this case can occur if $w<\frac{B+a-d}{2}$. If $w \vee a \geq \frac{B+a-d}{2}$, this case is suppressed. For the third case where $R \in\left(\frac{B+a-c}{2}, B\right]$, no consumer can win in NYOP auctions because the reserve price is too high. Similarly, there are multiple optimal reserve prices such that any value in $\left(\frac{B+a-c}{2} \vee w, B\right]$ is an optimal reserve price if $R^{*}>\frac{B+a-c}{2} \vee w$. This case must occur if $w>\frac{B+a-c}{2}$. Thus, the situation of multiple optimal reserve prices occurs when $R$ is too small or too big, because the retailer's profit function becomes independent of the reserve price. To be concise, in the sequel we ignore the multiplicity of the optimal reserve prices in the first and third cases.

\subsection{The Abundant Capacity Case}

Abundant capacity is a special case in which the NYOP channel has sufficient items. This situation might occur such as in off seasons when the consumer demand is weak. 
3.1.1. Single-Channel: NYOP Only. The case where the retailer has a single channel and abundant inventory capacity can be considered as a benchmark situation because of its simplicity. We first consider the case that $w<\frac{B+a-d}{2}$. If the retailer sets $R<\frac{B+a-d}{2}$, then every bid is higher than the reserve price, the expected profit from each bid is $\frac{B+a-\frac{1}{2}(c+d)}{2}$, and the retailer's expected profit is given by

$$
\Pi(R)=\frac{B+a-\frac{1}{2}(c+d)}{2}-w .
$$

The above $\Pi(R)$ is independent of $R$. Otherwise if $R \geq \frac{B+a-d}{2}$, the retailer's expected profit is given by

$$
\Pi(R)=\frac{B+a-2 R-c}{d-c}\left(\frac{\frac{B+a-c}{2}+R}{2}-w\right) .
$$

The above $\Pi(R)$ is concave in $R$, and the optimal solution is given by $R^{*}=w$. Since this result is obtained conditional on $w<\frac{B+a-d}{2}$, so any value in $\left[w, \frac{B+a-d}{2}\right)$ including $R^{*}=w$ can be an optimal reserve price. If $w \geq \frac{B+a-d}{2}$, since the case of $R<\frac{B+a-d}{2}$ is suppressed, the retailer can set $R^{*}=w$. Nevertheless, $w$ is an optimal reserve price for all situations. This result is intuitive because every qualified bid generates a positive profit for the retailer due to abundant capacity.

3.1.2. Dual-Channel: NYOP and List-Price. After the retailer adds a list-price channel, the optimal reserve price will change since some consumers who do not win in the NYOP channel will purchase directly from the list-price channel. We summarize the observations in the following proposition.

Proposition 3.1. In the single-bid dual-channel scenario with abundant capacity, $R^{*}=\hat{w}$, where $\hat{w}=w+\lambda(B-w)$.

Owing to the existence of a retailer-own list-price channel, the retailer sets a higher reserve price. The higher the portion of consumers who would purchase from the list-price channel, the higher the optimal reserve price. The value of $\lambda(B-w)$ is the expected profit from a customer whose bid is rejected from the NYOP auction. Since the retailer has abundant items, the retailer will not sell any item at a price below $\hat{w}=w+\lambda(B-w)$ in the NYOP channel. Indeed, we can regard $\hat{w}$ as the opportunity cost to the retailer for selling an item in the NYOP channel in a single-bid dual-channel scenario.

\subsection{The Constrained Capacity Case}

3.2.1. Single-Channel: NYOP Only. The number of total transactions in the constrained capacity case is $\min \left\{\frac{B+a-2 R-c}{d-c}, Q_{0}\right\}$ given that $(B+a-d) / 2 \leq R \leq$
$(B+a-c) / 2$. If $\frac{B+a-2 R-c}{d-c}<Q_{0}$, the situation is equivalent to the abundant capacity case. However, if $Q_{0} \leq \frac{B+a-2 R-c}{d-c}$, we have $\Pi(R)=Q_{0}\left(\frac{\frac{B+a-c}{2}+R}{2}-w\right)$. Hence, the optimal reserve price increases until it touches the upper boundary. Comparing the constrained capacity case with the abundant capacity case, we obtain the following result.

Proposition 3.2. In the single-bid single-channel scenario with constrained capacity, if $w \leq \frac{B+a-c-Q_{0}(d-c)}{2}$, then $R^{*}=\frac{B+a-c-Q_{0}(d-c)}{2} ;$ otherwise, $R^{*}=w$.

To explain Proposition 3.2 more intuitively, we illustrate the relation between $\Pi$ and $R$ in Figure 2 .

The concave quadratic curve is associated with the abundant capacity case given that $(B+a-d) / 2 \leq$ $R \leq(B+a-c) / 2$. The case of $w>\frac{B+a-c-Q_{0}(d-c)}{2}$ is associated with Line 1 in Figure 2, and the case of $w \leq \frac{B+a-c-Q_{0}(d-c)}{2}$ is associated with Line 2. If $w=\frac{B+a-c-Q_{0}(d-c)}{2}$, Lines 1 and 2 meet at $R=w$. From $w=\frac{B+a-c-Q_{0}(d-c)}{2}$, we obtain

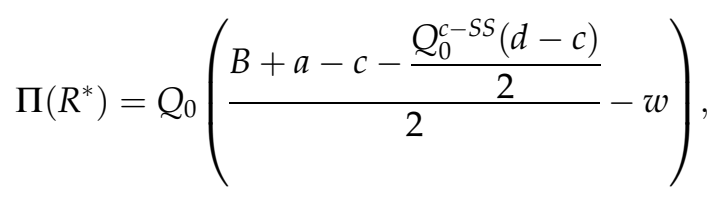

where

$$
Q_{0}^{c-S S}=\frac{B+a-c-2 w}{d-c} .
$$

The superscript SS represents single-bid singlechannel. We refer to $Q_{0}^{c}$ as the critical abundant capacity value throughout this paper. If the retailer has more inventory than the critical point (e.g., Line 1 in Figure 2), the retailer will set $R^{*}=w$; otherwise (e.g., Line 2 in Figure 2), the retailer will set $R^{*}=\frac{B+a-c-Q_{0}(d-c)}{2}$.

Figure 2 Relation Between $\Pi$ and $R$ in a Single-Bid Scenario SingleChannel Scenario with Constrained Capacity

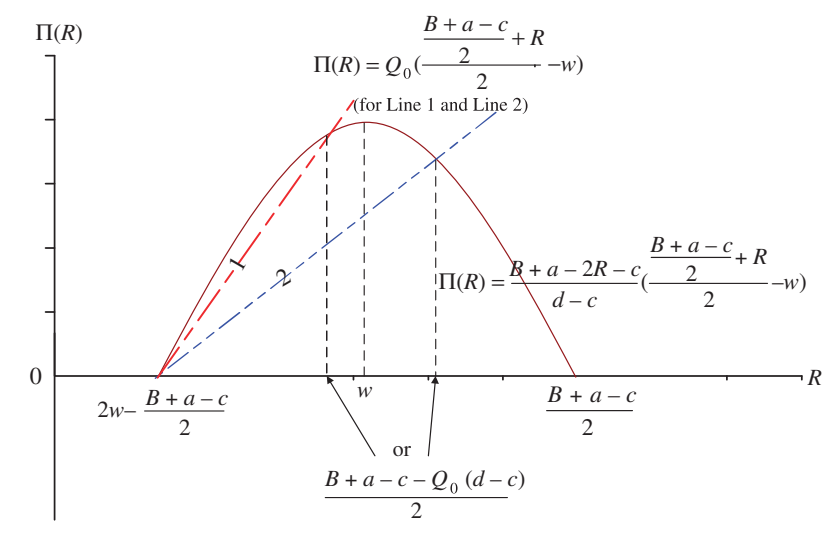


3.2.2. Dual-Channel: NYOP and List-Price. Similar to the dual-channel scenario with abundant capacity, unsatisfied customers from the NYOP auctions might switch to buy directly from the list-price channel. We have the following result.

Proposition 3.3. In the single-bid dual-channel scenario with constrained capacity, if $\hat{w} \leq \frac{1}{2}\left[B+a-c-Q_{0}(d-c)\right]$, $R^{*}=\frac{B+a-c-Q_{0}(d-c)}{2}$; otherwise, $R^{*}=\hat{w}$, where $\hat{w}=w+\lambda$ $(B-w)$.

Compare Proposition 3.3 with Proposition 3.2. The optimal reserve price is higher in the dual-channel scenario than in the single-channel scenario, if $\min \{\hat{w}, w\}>\frac{1}{2}\left[B+a-c-Q_{0}(d-c)\right]$ or equivalently $Q_{0}$ is big. If $Q_{0}$ is small such that $\max \{\hat{w}, w\} \leq$ $\frac{1}{2}\left[B+a-c-Q_{0}(d-c)\right]$, the optimal reserve prices are the same in both single-channel and dual-channel scenarios. Different from the abundant capacity case in Proposition 3.1, the optimal reserve price is determined by not only consumer preference to the list-price, but also the inventory capacity and ratio of unsatisfied consumers from the NYOP channel to buy from the retailer-own list-price channel.

Solving $\quad w+\lambda(B-w)=\frac{1}{2}\left[B+a-c-Q_{0}(d-c)\right]$, we obtain the critical abundant capacity value

$$
Q_{0}^{c-S D}=\frac{B+a-c-2(w+\lambda(B-w))}{d-c} .
$$

The superscript $S D$ represents single-bid dualchannel. We can infer that the higher $\lambda$, the lower $Q_{0}^{c-S D}$. We define $\lambda^{S D}=\frac{\frac{B+a-c}{2}-w}{B-w}$. When $\lambda \geq \lambda^{S D}$, we have $Q_{0}^{c-S D} \leq 0$. At this point, the retailer sets $R^{*}=$ $w+\lambda(B-w)$ and has no intention to sell products through the NYOP channel, but rather uses the NYOP as a mechanism to attract customers to buy from the retailer-own list-price channel. Whether to keep the NYOP channel or not will depend on whether it can attract customers to the retailer's store.

Comparing the dual-channel scenario with the single-channel scenario, we find that $Q_{0}^{c-S D} \leq Q_{0}^{c-S S}$, which suggests that the dual-channel retailer might want to limit the sale in the NYOP channel so as to obtain more profit from the list-price channel. In either scenario, the stronger consumer preference to the list-price, the smaller $Q_{0}^{c}$. If $Q_{0}>Q_{0}^{c-S S}$, then the optimal reserve price, i.e., $R^{*}=w+\lambda(B-w)$, is higher in the dual-channel than that, i.e., $R^{*}=w$, in the single-channel scenario. With a higher reserve price, the retailer can drive some consumers with low bids to buy from the retailer-own list-price channel. Otherwise if $Q_{0}<Q_{0}^{c-S D}$, then the optimal reserve price is the same, i.e., $R^{*}=\frac{B+a-c-Q_{0}(d-c)}{2}$, for both the single-channel and dual-channel scenarios. In both scenarios, the weaker the consumer pre- ference to list-price, the higher the optimal reserve price in the NYOP channel.

\section{A Double-Bid Scenario}

In this section we investigate the benefits of allowing the second bid. When focusing on a double-bid scenario similar to Fay (2004) and Spann et al. (2004), we introduce a different double-bid model. In our model, the consumer chooses an optimal bid $x^{*}$ for the first bid, and then adds a $\Delta$ dollar value to the second bid if the first bid fails. This schema is consistent with the recommendation by Segan (2005), who suggests that the consumer can add US $\$ 5$ to the previous bid, in addition to adding a new zone in rebidding a hotel on Priceline. This might also be close to real situations, in which consumers might determine how much more to rebid based on the initial bid, rather than re-compute the optimal bid. In line with the literature, such as Spann et al. (2004), we assume that the consumers are rebidding on the same item and their preferences remain the same during the bidding. The consumer's initial belief about the winning probability of bidding $x$ is the same as in the single-bid scenario; however, the consumer will update the belief if the consumer fails to win with the initial bid. We denote the new belief CDF by $F_{2}$ and the corresponding PDF by $f_{2}$. Thus,

$$
F_{2}(x+\Delta)=\frac{x+\Delta-x}{B-x}=\frac{\Delta}{B-x},
$$

and

$$
f_{2}(x+\Delta)=\frac{\Delta}{(B-x)^{2}} .
$$

The consumer aims to minimize the cost, given that the consumer will switch to buy from the list-price channel if he/she fails both bids. The objective function is

$$
\begin{aligned}
\min _{x, \Delta} C(\theta)= & F(x)(x+\theta)+(1-F(x)) \\
& {\left[F_{2}(x+\Delta)(x+\Delta+\theta)\right.} \\
& \left.+\left(1-F_{2}(x+\Delta)\right) B\right],
\end{aligned}
$$

where the first term is the expected cost of winning the item at $x$, and the second term is the expected cost of not winning the first bid but winning the second bid, or buying from the list-price channel after failing both bids. The optimal double-bid strategy is given as follows.

Proposition 4.1. In the double-bid scenario, the optimal initial bid for the consumer is

$$
x^{*}=\frac{B+a-\theta-\Delta^{*}}{2},
$$


and the optimal second bid is $x^{*}+\Delta^{*}$, where

$$
\Delta^{*}=\frac{1}{3}(B-a-\theta)
$$

As compared with the single-bid scenario, a consumer first bids $\Delta^{*} / 2$ lower and then bids $\Delta^{*} / 2$ higher than the optimal bid in the single-bid scenario. Furthermore, substituting the optimal bids into the objective function, we can show that a double-bid scenario is a better choice for the consumer as long as $\Delta^{*}<\frac{2}{3}(B-a-\theta)$, which is true in Proposition 4.1. Thus, everything else being equal, a double-bid scenario could save costs for the consumers. As a result, a double-bid scenario can be more attractive than the single-bid scenario to the consumers.

\subsection{Single-Channel: NYOP only}

The retailer accepts bids higher than the reserve price; hence, in the consumer's optimal bidding strategy, the first bid is accepted if and only if $\theta \leq B+2 a-3 R$. As for the second bid, $x^{*}+\Delta^{*}=\frac{B+a-\theta+\Delta^{*}}{2} \geq R$ is equivalent to $\theta \leq B+\frac{1}{2} a-\frac{3}{2} R$. For a given $R$, we must have $B+2 a-3 R \leq B+\frac{1}{2} a-\frac{3}{2} R$. Based on the consumer preference boundary values, we may divide consumers into the following categories. To avoid overlapping in the following cases, we assume that $B-a<2 d-c$. This assumption is consistent with the fact that consumer preference toward Priceline's NYOP auction is quite diversified such that $d$ could be a value close to $B$ and $c$ could be a value close to 0 . This is further supported by the observation that some customers never shop in the NYOP channel even if Priceline promises up to $50 \%$ off the regular price in the NYOP channel.

Case 1: $d \leq B+2 a-3 R$, equivalently $R \leq R_{3}=\frac{B+2 a-d}{3}$. In this case consumers very weakly prefer the listprice channel, and every customer wins an item from the NYOP channel with the first bid.

Case 2: $c \leq B+2 a-3 R \leq d \leq B+\frac{1}{2} a-\frac{3}{2} R$, equivalently $R_{3}<R \leq R_{4}=\frac{2 B+a-2 d}{3}$. In this case the consumers weakly prefer the list-price channel, and every customer wins an item from the NYOP channel with either the first or second bid.

Case 3: $c \leq B+2 a-3 R \leq B+\frac{1}{2} a-\frac{3}{2} R \leq d$, equivalently $R_{4}<R \leq R_{5}=\frac{B+2 a-c}{3}$. In this case the consumers fairly prefer the list-price channel, and some customers win items from the NYOP channel with either the first or second bid.

Case 4: $\quad B+2 a-3 R \leq c \leq B+\frac{1}{2} a-\frac{3}{2} R$, equivalently $R_{5}<R \leq R_{6}=\frac{2 B+a-2 c}{3}$. In this case the consumers strongly prefer the list-price channel, and thus every customer's first bid is rejected while some second bids are accepted.

Case 5: $B+\frac{1}{2} a-\frac{3}{2} R<c$, or $R>R_{6}$. In this case the consumers very strongly prefer the list-price channel, and no customer wins from the NYOP channel.

The above cases are illustrated in Scenario A of Figure 3. Note that Scenario $A$ is for the abundant capacity case, while Scenarios B, C, and D are for the constrained capacity case. Notation of $\hat{Q}_{1}$ and $\hat{Q}_{2}$ is further described in Equations (6) and (7).

To obtain tractability, we assume that the first bid has priority over the second bid to be satisfied in this double-bid scenario. Although it is reasonable for the retailer to sell items to higher bids, this assumption can be considered as a tie-breaking rule. This rule has been adopted in some online auction web sites, e.g., uBid.com, to encourage consumers to bid early. Let $D_{1}$ and $D_{2}$ be the demand to the NYOP channel from the first and second bids, respectively.

$$
\begin{aligned}
D_{1} & =P\left\{x^{*} \geq R\right\}=P\left\{\frac{B-\theta+2 a}{3} \geq R\right\} \\
& =P\{\theta \leq B+2 a-3 R\}, \\
D_{2} & =P\left\{x^{*}+\Delta^{*} \geq R\right\}-P\left\{x^{*} \geq R\right\} .
\end{aligned}
$$

For the above Cases 1-5, we have

$$
\begin{aligned}
& \left(1, \quad \text { if } R \in\left[w \vee a, \frac{B+2 a-d}{3}\right)\right. \text {; } \\
& D_{1}= \begin{cases}\frac{B+2 a-3 R-c}{d-c}, & \text { if } R \in\left[\frac{B+2 a-d}{3} \vee w, \frac{2 B+a-2 d}{3}\right) ; \\
\frac{B+2 a-3 R-c}{d-c}, & \text { if } R \in\left[\frac{2 B+a-2 d}{3} \vee w, \frac{B+2 a-c}{3}\right) ; \\
0, & \text { if } R \in\left[\frac{B+2 a-c}{3} \vee w, \frac{2 B+a-2 c}{3}\right) ;\end{cases} \\
& \text { 0, } \quad \text { if } R \in\left[\frac{2 B+a-2 c}{3} \vee w, B\right] . \\
& \text { (0, } \quad \text { if } R \in\left[w \vee a, \frac{B+2 a-d}{3}\right) \text {; } \\
& 1-\frac{B+2 a-3 R-c}{d-c}, \text { if } R \in\left[\frac{B+2 a-d}{3} \vee w, \frac{2 B+a-2 d}{3}\right) ; \\
& D_{2}=\left\{\frac{\frac{3}{2}(R-a)}{d-c}, \quad R \in\left[\frac{2 B+a-2 d}{3} \vee w, \frac{B+2 a-c}{3}\right) ;\right. \\
& \begin{array}{ll}
\frac{B+\frac{1}{2} a-\frac{3}{2} R-c}{d-c}, & \text { if } R \in\left[\frac{B+2 a-c}{3} \vee w, \frac{2 B+a}{3}\right. \\
0, & \text { if } R \in\left[\frac{2 B+a-2 c}{3} \vee w, B\right] .
\end{array}
\end{aligned}
$$

Similar to the single-bid scenario, there will be multiple optimal reserve prices if $w>\frac{2 B+a-2 c}{3}$. For the same reason, we skip the discussion on the multiplicity of the optimal reserve prices. 
Figure 3 Case Categories in Terms of Reserve Price and Resource Capacity When $\hat{Q}_{1}<\hat{Q}_{2}$

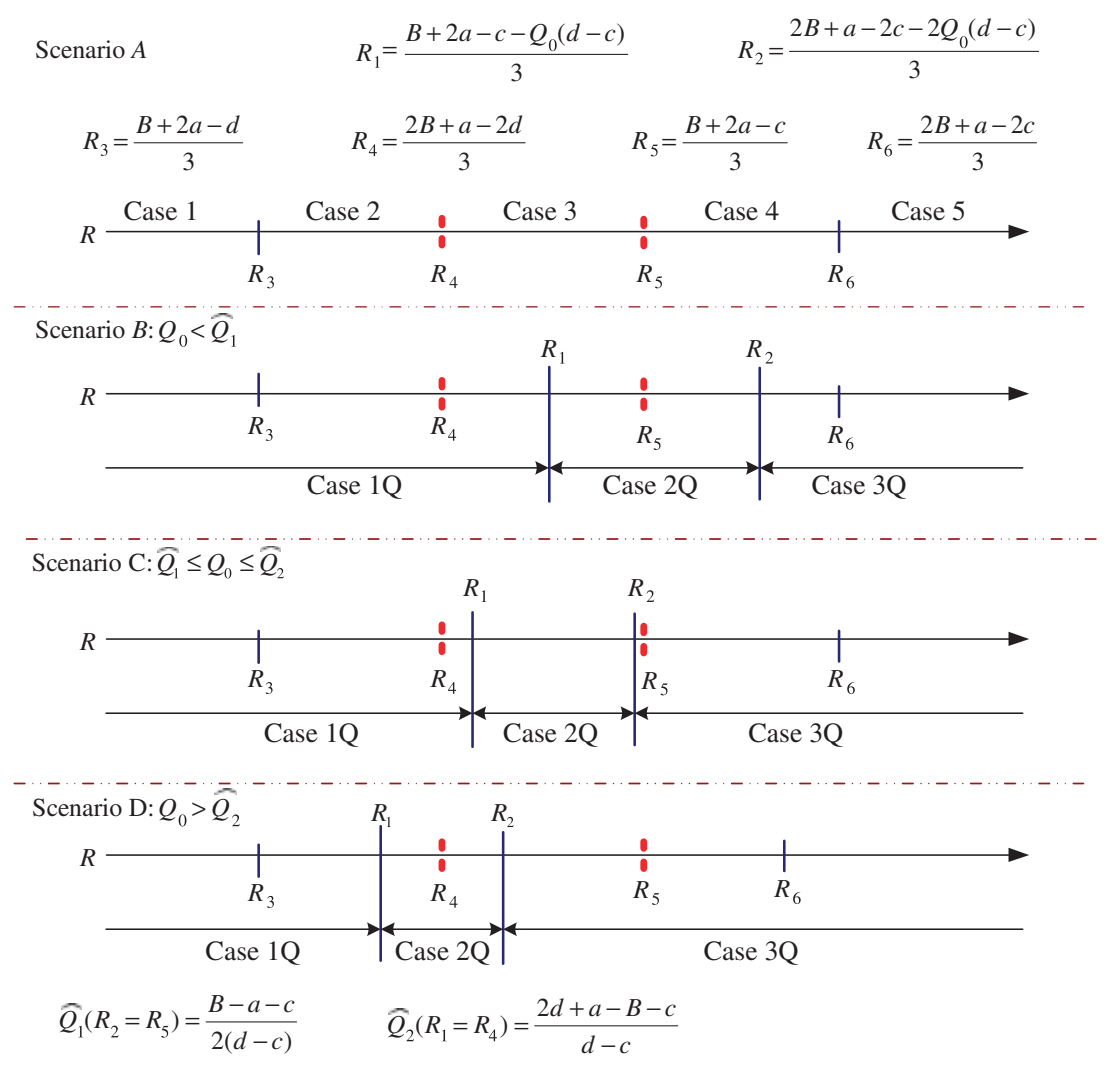

For the abundant capacity case, we have the following observation.

PROPOSITION 4.2. In the double-bid single-channel scenario with abundant capacity, the optimal reserve price is given by

$$
\begin{cases}\text { if } w<\frac{2 d+9 a+c-3 B}{6}, & R^{*}=\frac{2 B+a-2 d}{3}, \\ \text { if } \frac{2 d+9 a+c-3 B}{6} \leq w \leq \frac{B+2 a-c}{3}, & R^{*}=\frac{B+2 a-c}{3} \\ \text { if } w>\frac{B+2 a-c}{3}, & R^{*}=w .\end{cases}
$$

Proposition 4.2 is conditional on $\frac{2 d+9 a+c-3 B}{6}>0$. However, if $\frac{2 d+9 a+c-3 B}{6}<0$, Equation (5) can be rewritten as $R^{*}=\max \left\{\frac{B^{6}+2 a-c}{3}, w\right\}$. Overall, Proposition 4.2 shows that the optimal reserve price is increasing step-wisely with respect to the wholesale price. As compared with Proposition 3.1, Proposition 4.2 shows that the optimal reserve price in the double-bid scenario is higher than that in the single-bid scenario if $\frac{B+2 a-c}{3}>w$. This occurs because the retailer wants to avoid some extremely low bids because of a more lenient policy.

We now consider the constrained capacity case. If $Q_{0} \leq \frac{B+2 a-3 R-c}{d-c}$, i.e., $R \leq R_{1}=\frac{B+2 a-c-Q_{0}(d-c)}{3}$, the limited capacity can only satisfy partial demand of the first bids. If $Q_{0} \leq \frac{B+\frac{1}{2} a-\frac{3}{2} R-c}{d-c}$, i.e., $R \leq R_{2}=\frac{2 B+a-2 c-2 Q_{0}(d-c)}{3}$, the limited capacity can only satisfy partial demand of the second bids. $R_{1}$ and $R_{2}$ are illustrated in Scenarios $\mathrm{B}, \mathrm{C}$, and D of Figure 3. Comparing $R_{1}, R_{2}$ with $R_{3}, R_{4}$, $R_{5}, R_{6}$, we can infer that $R_{1}$ will be between $R_{3}$ and $R_{5}$, and $R_{2}$ will be between $R_{4}$ and $R_{6}$. However, the relative positions between $R_{1}$ and $R_{4}$ and between $R_{2}$ and $R_{5}$ are determined by the capacity level $Q_{0}$. Let $R_{2}=R_{5}$, we obtain

$$
\hat{Q}_{1}=\frac{B-a-c}{2(d-c)} .
$$

Similarly letting $R_{1}=R_{4}$ gives us

$$
\hat{Q}_{2}=\frac{2 d+a-B-c}{d-c} .
$$

We categorize $Q_{0}$ into different scenarios as illustrated in Figure 3 when $\hat{Q}_{1}<\hat{Q}_{2}$. In Scenario $B, Q_{0}$ satisfies all first bids in Case 3 and all second bids in Case 4. If $Q_{0}$ increases, $R_{1}$ and $R_{2}$ move up to Case 3 in Scenario $C$ and then to Case 2 and Case 3, respectively, in Scenario D. We analyze the above situations in more detail and obtain the following proposition. 
PROPOSITION 4.3. In the double-bid single-channel scenario with constrained capacity, if $Q_{0}<\hat{Q}_{1}$, $R^{*}=\max \left\{\frac{2 B+a-2 c-2 Q_{0}(d-c)}{3}, w\right\} ;$ otherwise,
We now consider the special case that $Q_{0}<\hat{Q}_{1}$ and $w$ is small enough such that $\frac{2 B+a-2 c-2 Q_{0}(d-c)}{3}$ is the global optimal reserve price. We can prove that

$$
\begin{cases}\text { if } w<\frac{9 a-3 B+c+2 d-2\left(1-Q_{0}\right)(d-c)}{6}, & R^{*}=\frac{2 B+a-2 c-2 Q_{0}(d-c)}{3} \\ \text { if } \frac{9 a-3 B+c+2 d-2\left(1-Q_{0}\right)(d-c)}{6} \leq w \leq \frac{B+2 a-c}{3}, & R^{*}=\frac{B+2 a-c}{3}, \\ \text { if } w>\frac{B+2 a-c}{3}, & R^{*}=w .\end{cases}
$$

Comparing Proposition 4.3 with Proposition 4.2 shows that the optimal reserve price is set to the wholesale price if the wholesale price is high. If the wholesale price is low such that $w<\frac{9 a-3 B+c+2 d-2\left(1-Q_{0}\right)(d-c)}{6}$, the retailer will use a higher reserve price than that in the abundant capacity case as shown in Proposition 4.2. We partially demonstrate Propositions 4.2 and 4.3 in the following example.

EXAMPLE 1. Suppose that the constrained capacity $Q_{0}=0.6$. The list-price is $B=100$. The consumer preference is on $[c, d]=[0,60]$. The winning probability is on $[a, B]=[35,100]$. The wholesale price is $w=20$. Hence, we obtain $\hat{Q}_{1}=54.17$ and $\hat{Q}_{2}=91.67$ and it is belonging to Scenario $C$, i.e., $\hat{Q}_{1}<Q_{0}<\hat{Q}_{2}$. Two curves of Cases 1-4 and 1Q-3Q as shown in the proofs of Propositions 4.2 and 4.3 for a double-bid situation can be described in Figure 4 . The optimal reserve price is given by $R_{5}=56.67$. The corresponding profit is $\Pi_{\text {Double-Bid }}^{*}=25.73$. In contrast, for the single-bid scenario, the optimal reserve price is given by $R^{*}=49.5$ and the optimal profit is $\Pi_{\text {Single-Bid }}^{*}=23.1$. This empirically shows that the double-bid can outperform the single-bid, i.e., the retailer's profit is higher in the double-bid case.

Figure 4 Relation Between $\Pi$ and $R$ in a Double-Bid Single-Channel Scenario with $\hat{Q}_{1}<\mathbf{Q}_{0}<\hat{\mathbf{Q}}_{2}$

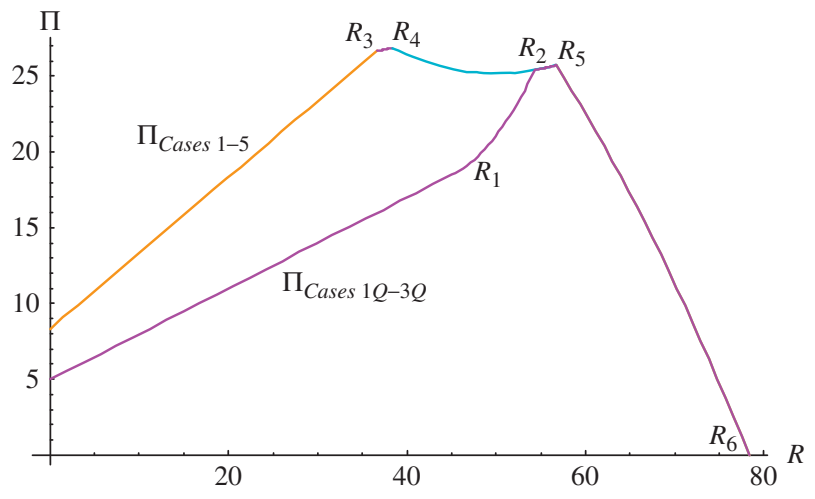

$\frac{2 B+a-2 c-2 Q_{0}(d-c)}{3} \geq \frac{B+a-c-Q_{0}(d-c)}{2}$ given that $B-a \geq d$. Thus, the optimal reserve price is more likely to be higher in the double-bid scenario than in the singlebid scenario. This occurs especially when $Q_{0}$ is small and the retailer wants to avoid selling the products to low bids. In this special case, the optimal profit is given by

$$
\begin{aligned}
& \Pi\left(R^{*}\right) \\
& =Q_{0}\left(\frac{B+\frac{1}{2} a+\frac{3}{2}\left(\frac{2 B+a-2 c-2 Q_{0}(d-c)}{3}\right)-c}{3}-w\right) .
\end{aligned}
$$

Taking the first-order derivative on $\Pi\left(R^{*}\right)$ with respect to $Q_{0}$ yields the critical abundant capacity value,

$$
Q_{0}^{c-D S}=\frac{2 B+a-2 c-3 w}{2(d-c)} .
$$

The superscript DS represents double-bid singlechannel. Comparing this profit with the profit of the single-bid scenario when $Q_{0}<Q_{0}^{c-S S}$, we have $\Pi_{\text {Double-Bid }}^{*}-\Pi_{\text {Single-Bid }}^{*}=\frac{Q_{0}(d-c)}{12}\left(\frac{2(B-a-c)}{d-c}-Q_{0}\right) \geq 0$ given that $Q_{0} \leq \hat{Q}_{1}<\frac{2(B-a-c)}{d-c}$. Thus, we obtain the following result.

PROPOSITION 4.4. In the single-channel scenario, if $Q_{0}<$ $\min \left\{Q_{0}^{c-D S}, Q_{0}^{c-S S}, \hat{Q}_{1}\right\}$, then the double-bid scenario outperforms the single-bid scenario, i.e., the retailer receives higher profit when offering two bidding options.

Proposition 4.4 indicates that if the inventory capacity is small satisfying the above condition, the double-bid is a better policy than the single-bid. The intuition lies in the consumer behavior difference in the single-bid and double-bid scenarios. Because the consumers' second bids in the double-bid scenario are higher than the bids in the single-bid scenario, the retailer can design a higher reserve price in the double-bid scenario to reject the first bids and accept the second bids instead. This is supported by the 
Figure 5 The Optimal Profit Comparison Between Single-Bid and DoubleBid in Terms of $Q_{0}$ with $w=10$

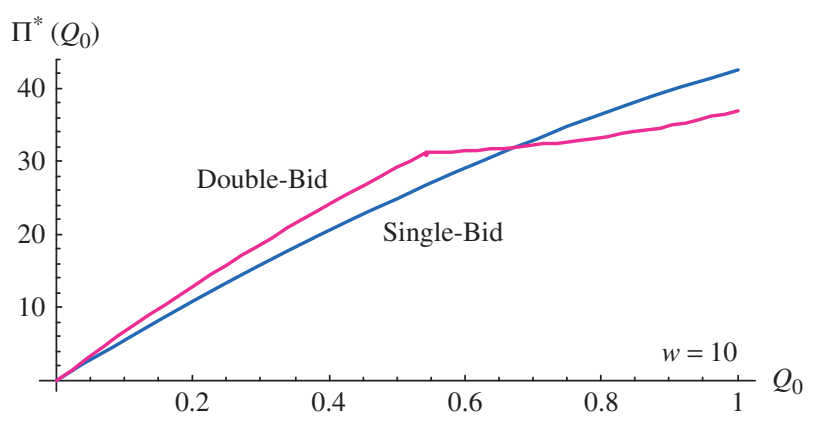

comparison of the optimal reserve prices in those two scenarios. The following is true:

$$
R_{\text {Double-Bid }}^{*}-R_{\text {Single-Bid }}^{*}=\frac{B-a-c-Q_{0}(d-c)}{6}>0
$$

as long as $Q_{0}<\frac{B-a-c}{d-c}$, which holds given that $Q_{0}<\hat{Q}_{1}=$ $\frac{B-a-c}{2(d-c)}$.

It is worth noting that Proposition 4.4 is independent of the previous assumption that the first bids have the priority. As we show in the proof of Proposition 4.3, $Q_{0}<\min \left\{Q_{0}^{c-D S}, Q_{0}^{c-S S}, \hat{Q}_{1}\right\}$ corresponds to the case that all first bids are rejected and some second bids are accepted; thus, Proposition 4.4 is robust against the first-bid priority.

The computational complexity of cases that $Q_{0}>\hat{Q}_{1}$ prevents us from obtaining meaningful analytic results. Instead, we numerically compare the optimal profits in the single-bid and double-bid scenarios as illustrated in Figures 5 and 6. The market configuration is the same as in Example 1, except that the wholesale price is changed to $w=10$ and 40, respectively, in two separate cases. Figures 5 and 6 further support Proposition 4.4, which states that the doublebid scenario outperforms the single-bid scenario when $Q_{0}$ is small regardless of the value of the wholesale price. However, Figure 5 illustrates that if the wholesale price is low, i.e., $w=10$, the single-bid scenario
Figure 6 The Optimal Profit Comparison Between Single-Bid and DoubleBid in Terms of $Q_{0}$ with $w=40$

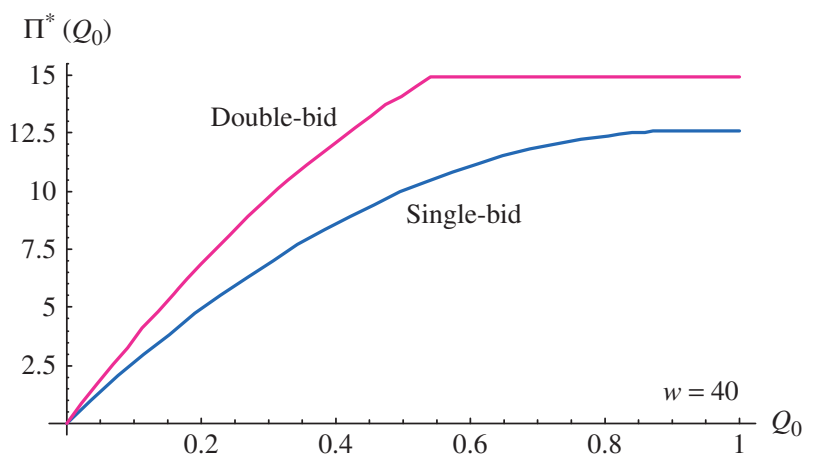

to sell more opaque products, and the low wholesale price provides enough cushion for the retailer to lower the reserve price in the double-bid scenario to catch some first bids. But, by doing so, the retailer loses some profits due to those first bids as compared with that in the single-bid scenario. If the wholesale price is higher, i.e., $w=40$, the double-bid scenario outperforms the single-bid scenario for all values of $Q_{0}$. A high wholesale price does not give much flexibility for both single-bid and double-bid scenarios. As shown in Propositions 3.2 and 4.3, the optimal reserve price becomes close to the wholesale price especially when $Q_{0}$ grows big. In this situation, the double-bid scenario has an advantage over the singlebid scenario; since more first bids fail due to the high wholesale price and corresponding high reserve price, the retailer benefits from more second bids.

\subsection{Dual-Channel: NYOP and List-Price}

In the dual-channel scenario, a portion of unsatisfied bidders switch to buy from the retailer-own list-price channel. Similarly to the single-channel situation, we have the following result.

Proposition 4.5. In the double-bid dual-channel scenario with constrained capacity, if $Q_{0}<\hat{Q}_{1}$, then $R^{*}=$ $\max \left\{\frac{2 B+a-2 c-2 Q_{0}(d-c)}{3}, \hat{w}\right\} ;$ otherwise,

$$
\begin{cases}\text { if } \hat{w}<\frac{9 a-3 B+c+2 d-2\left(1-Q_{0}\right)(d-c)}{6}, & R^{*}=\frac{2 B+a-2 c-2 Q_{0}(d-c)}{3}, \\ \text { if } \frac{9 a-3 B+c+2 d-2\left(1-Q_{0}\right)(d-c)}{6} \leq \hat{w} \leq \frac{B+2 a-c}{3}, & R^{*}=\frac{B+2 a-c}{3}, \\ \text { if } \hat{w}>\frac{B+2 a-c}{3}, & R^{*}=\hat{w},\end{cases}
$$

outperforms the double-bid scenario when $Q_{0}$ is big, e.g., $Q_{0}>0.65$ approximately in this example. This occurs because when $Q_{0}$ is big, the retailer needs where $\hat{w}=w+\lambda(B-w)$.

Conclusion from Proposition 4.5 is similar to Proposition 4.3, except that $\hat{w}=w+\lambda(B-w)>w$ as long 
as $\lambda>0$. The retailer will more likely charge a higher reserve price. The higher the percentage of consumers that switch to the retailer-own list-price channel, the higher the optimal reserve price.

Similar to the single-channel scenario when $Q_{0}<\hat{Q}_{1}$ and $\hat{w}$ is small enough such that $\frac{2 B+a-2 c-2 Q_{0}(d-c)}{3}$ is the global optimal reserve price, the optimal profit is given by

$$
\begin{aligned}
\Pi\left(R^{*}\right)= & Q_{0}\left(\frac{B+\frac{1}{2} a+\frac{3}{2}\left(\frac{2 B+a-2 c-2 Q_{0}(d-c)}{3}\right)-c}{3}-w\right) \\
& +\lambda\left(1-Q_{0}\right)(B-w)-C_{f} .
\end{aligned}
$$

Taking the first-order derivative on $\Pi\left(R^{*}\right)$ with respect to $Q_{0}$ yields the critical abundant capacity value,

$$
Q_{0}^{c-D D}=\frac{2 B+a-2 c-3 \hat{w}}{2(d-c)},
$$

where the superscript $D D$ represents double-bid dualchannel. We summarize the critical abundant capacity values in Table 1 . We have $Q_{0}^{c-D D} \leq Q_{0}^{c-D S}$, which suggests that the dual-channel retailer is more motivated than in the single-channel scenario, to drive some NYOP customers to buy from the retailer-own list-price channel. We define $\lambda^{D D}=\frac{\frac{2 B+a-2 c}{3}-w}{B-w}$. When $\lambda \geq \lambda^{D D}$, we have $Q_{0}^{c-D D} \leq 0$, where similar to that in the single-bid scenario, the retailer will charge a reserve price at $w+\lambda(B-w)$ with no intention to sell products through the NYOP channel. Rather the retailer uses the NYOP as a mechanism to attract customers to buy from the retailer-own list-price channel. In addition, we can show that $\lambda^{D D}-\lambda^{S D}=$ $\frac{B-a-c}{6}>0$, which indicates that the retailer may be more willing to sell through the NYOP channel in the double-bid scenario than in the single-bid scenario when $\lambda$ is a high value.

Similar to Proposition 4.4, we have the following result.

Proposition 4.6. In the dual-channel scenario, if $Q_{0}<\min \left\{Q_{0}^{c-D D}, Q_{0}^{c-S D}, \hat{Q}_{1}\right\}$, then the double-bid scenario outperforms the single-bid scenario.

Owing to our special focus on finding the optimal reserve prices, the dual-channel advantage will largely depend on the cost structure by adding a

Table 1 Critical Abundant Capacity Values $Q_{0}^{c}$

\begin{tabular}{lll}
\hline & \multicolumn{1}{c}{ Single-bid } & \multicolumn{1}{c}{ Double-bid } \\
\hline Single-channel & $\frac{B+a-c-2 w}{d-c}$ & $\frac{2 B+a-2 c-3 w}{2(d-c)}$ \\
Dual-channel & $\frac{B+a-c-2(w+\lambda(B-w))}{d-c}$ & $\frac{2 B+a-2 c-3(w+\lambda(B-w))}{2(d-c)}$ \\
\hline
\end{tabular}

list-price channel. Here we use the following example to partially illustrate the above discussion.

ExAmple 2. We continue from Example 1. Suppose that $w=40$ and $C_{f}=500$. Since $R_{5}=56.67>40=w, R^{*}=$ 56.67 in the single-channel scenario. In the dualchannel situation, we first assume that the portion of unsatisfied customers from the NYOP auctions to the retailer-own list-price channel is given by $\lambda=0.2$. We have $\hat{w}=w+\lambda(B-w)=52<56.67=R_{5}$, and hence the optimal price continues to be $R^{*}=R_{5}$ and $\Pi(R)^{*}=$ 15.40. However, if $\lambda=0.3$, we have $\hat{w}=58>$ $56.67=R_{5}$, and thus, the new optimal reserve price becomes $R^{*}=\hat{w}=58$ and $\Pi(R)^{*}=18.17$. In the single-channel scenario, $\Pi(R)^{*}=16.50$. So, the singlechannel scenario outperforms the dual-channel scenario if $\lambda=0.2$; however, the dual-channel scenario outperforms the single-channel scenario if $\lambda=0.3$. This implies that it is important for the retailer to retain the unsatisfied customers from the NYOP auctions so they will purchase from the retailer-own list-price channel. On the other hand, given that $\lambda$ is constant, it is a plausible approach to attract more customers to the retailer-own list-price channel via NYOP auctions. Thus, the double-bid policy may become more significant if it can attract more customers to the retailer-own list-price channel.

\section{Conclusion}

This paper evaluates the optimal reserve prices in the NYOP channel with bidding options in the presence of list-price channels. We investigate a single-bid scenario and a double-bid scenario, in which the consumers can bid twice in the NYOP channel, and provide insights into whether the double-bid scenario can outperform the single-bid scenario. We also study the impact of adding a retailer-own list-price channel on the optimal reserve prices. The analysis of this paper may facilitate the understanding of determining the optimal reserve price in a variety of situations and help retailers further revise their operation mechanism.

In both the single-bid and double-bid scenarios, the optimal reserve price is higher when the inventory capacity is limited than when the inventory capacity is abundant. The optimal reserve price is higher or at least not lower in the double-bid scenario than that in the single-bid scenario. After adding an NYOPretailer-own list-price channel, the optimal reserve price remains the same if the inventory capacity is low, but could increase if the inventory capacity becomes high.

In both single-channel and dual-channel scenarios, the lower the consumer preference to the list-price channel (or the higher the consumer preference to the NYOP channel), the higher the optimal reserve price in the NYOP channel. If the portion of customers 
who do not win in the NYOP channel but purchase in the retailer-own list-price channel is high enough, the retailer will charge a high reserve price with no intention to sell the products through the NYOP channel, but rather use it as a marketing tool to attract customers to buy from the retailer-own listprice channel.

The double-bid scenario can outperform the singlebid scenario in both the single-channel and dualchannel scenarios. If the NYOP inventory capacity is low, we show that the double-bid scenario outperforms the single-bid scenario. However, if the wholesale price is low, the single-bid scenario can outperform the double-bid scenario when the inventory capacity grows. In practice, if a double-bid policy can attract more consumers to the dual-channel retailer, the retailer might want to consider the doublebid scenario more seriously in order to bring more customers to the list-price channel.

This paper has its limitations. First, although the assumption of uniform distribution functions is common in the literature, it is desirable to relax this assumption to obtain more insights. For example, in the double-bid scenario, the symmetric result, where the average of the two bids in the double-bid scenario equals the bid in the single-bid scenario, will likely be altered with an asymmetry distribution function. Second, in the double-bid scenario, the assumption that the first bids have priority is made to achieve tractability but might be too strong. As we argue that the first-bid priority might encourage the consumers to bid early, it is reasonable to assume that the retailer will sell items to higher bids. Some simulation tools can be utilized to examine the impact of different tiebreaking rules. Third, due to the special focus of this paper, the list-channels owned by other sellers are not game theoretic. Theoretically and practically, the retailer may dynamically compete with other listprice channels. As a result, the analysis of adding a list-price channel will be more complicated than that of the simplified setting in this paper. Since the consumer behavior in this paper depends on the interaction between the NYOP channel and those listprice channels, it becomes computationally intractable to consider the retail-level multi-channel competition in addition to the consumer NYOP bidding behavior. However, it is worth exploring whether we can analyze the strategic perspective of multi-channel competition by simplifying the consumer behavior in the NYOP channel. Fourth, the consumer belief function is relatively static in the model. As shown in Priceline's history, how to influence the consumer bidding behavior has been an intriguing practical issue. Last, but not the least, extension to considering stochastic entrance of consumers will be another new avenue for future work.

\section{Acknowledgments}

The authors are grateful to the Senior Editor and the two anonymous reviewers for their comments and suggestions, which have helped significantly improve the quality of this paper. The authors are also indebted to Matthew Winger, Dacheng Yao, Guoren Zhang, and Hanqin Zhang for their very valuable inputs.

\section{Appendix}

Proof of Proposition 3.1. We analyze this scenario when $w>a$. We start from the condition that $w<(B+a-d) / 2$. We consider three cases separately.

Case 1: If $R<(B+a-d) / 2$, or equivalently $d<B+a-2 R$, every customer wins an item from the retailer's NYOP auction. The retailer's expected profit is given by

$$
\Pi(R)=\left[\frac{B+a-\frac{1}{2}(c+d)}{2}-w\right]-C_{f} .
$$

The profit is independent of the reserve price.

Case 2: If $(B+a-d) / 2 \leq R \leq(B+a-c) / 2$, or equivalently $d \geq B+a-2 R \geq c$, some consumers win from the NYOP auction, and the retailer's expected profit is given by

$$
\begin{aligned}
\Pi(R)= & \frac{B+a-2 R-c}{d-c}\left(\frac{\frac{B+a-c}{2}+R}{2}-w\right) \\
& +\lambda\left(1-\frac{B+a-2 R-c}{d-c}\right)(B-w)-C_{f} .
\end{aligned}
$$

$\Pi(R)$ is concave in $R$. The first-order derivative is

$$
\frac{\partial \Pi(R)}{\partial R}=\frac{2 w+2 \lambda(B-w)-2 R}{d-c} .
$$

Thus, the optimal reserve price is $R^{*}=\hat{w}=w+$ $\lambda(B-w)$. The optimal expected profit is

$$
\begin{aligned}
\Pi(R)= & \frac{B+a-2(w+\lambda(B-w))-c}{d-c} \\
& \times\left(\frac{\frac{B+a-c}{2}+w+\lambda(B-w)}{2}-w\right) \\
& +\lambda\left(1-\frac{B+a-2(w+\lambda(B-w))-c}{d-c}\right) \\
& \times(B-w)-C_{f} .
\end{aligned}
$$


Case 3: If $R>(B+a-c) / 2$, or equivalently $B+a-2 R<c$, no consumer wins from the NYOP auction. Thus, the retailer's expected profit is given by

$$
\Pi(R)=\lambda(B-w)-C_{f}
$$
well.

The profit is independent of the reserve price as

Combining the above three cases, we have

1. If $(B+a-d) / 2 \leq \hat{w} \leq(B+a-c) / 2$, then $R^{*}=\hat{w}$, and the optimal profit is given by Equation (A1).

2. If $\hat{w}<(B+a-d) / 2$, then the optimal reserve price can be any value in $[w,(B+a-d) / 2)$ including $\hat{w}$.

3. If $\hat{w}>(B+a-c) / 2$, then the optimal reserve price can be any value in $[(B+a-c) / 2), B-a)$ including $\hat{w}$, where $R \leq B-a$ means that there is a non-negative possibility that some customers would buy in the NYOP channel.

Thus, in all above situations, $\hat{w}$ is the optimal reserve price.

When $w$ grows such that $(B+a-d) / 2<w<$ $(B+a-c) / 2$, Case 1 is suppressed. If $w>(B+a-c) / 2$, Cases 1 and 2 are suppressed. Since $a<(B+a-d) / 2$, the case of $w \leq a$ is limited to the first case of the demand function where $D_{N Y O P}=1$ and can be analyzed similarly. In all situations, the result that $\hat{w}$ is the optimal reserve price holds.

ProOF OF Proposition 3.2. If $Q_{0}>\frac{B+a-2 R-c}{d-c}$, then the retailer's profit is given by $\Pi(R)_{1}=$ $\frac{B+a-2 R-c}{d-c}\left(\frac{\frac{B+a-c}{2}+R}{2}-w\right)$, which is a concave quadratic curve. If $Q_{0} \leq \frac{B+a-2 R-c}{d-c}$, then the retailer's profit becomes $\Pi(R)_{2}=Q_{0}\left(\frac{\frac{B+a-c}{2}+R}{2}-w\right)$ (Lines 1 or 2 as illustrated in Figure 2). The retailer's profit function curve is first represented by $\Pi(R)_{2}$ and then $\Pi(R)_{1}$. The interception point of $\Pi(R)_{2}$ and $\Pi(R)_{1}$ is given by $R=\frac{B+a-c-Q_{0}(d-c)}{2}$. If $\frac{B+a-c-Q_{0}(d-c)}{2} \geq w$, then $\Pi(R)_{2}$ and $\Pi(R)_{1}$ intercept after the point $R=w$ and thus $R^{*}=\frac{B+a-c-Q_{0}(d-c)}{2}$. The corresponding expected profit is given by $\Pi\left(R^{*}\right)=Q_{0}\left(\frac{B+a-c-\frac{Q_{0}(d-c)}{2}}{2}-w\right)$. Otherwise if $R=\frac{B+a-c-Q_{0}(d-c)}{2}<w, \Pi(R)_{2}$ and $\Pi(R)_{1}$ intercept before the point $R=w$ and thus the unique global maximum point is obtained at $R^{*}=w$, and we have the optimal expected profit $\Pi\left(R^{*}\right)=\frac{(B+a-c-2 w)^{2}}{4(d-c)}$.

Proof of Proposition 3.3. The analysis of Proposition 3.3 will be a combination of Propositions 3.1 and 3.2. We only consider the situation that $w<(B+a-d) /$ $2<R<(B+a-c) / 2$, and the analysis of other situations will be similar. The number of customers that win an item from NYOP is given by $\min \left\{\frac{B+a-2 R-c}{d-c}, Q_{0}\right\}$. Similarly, we consider two cases as follows.

Case 1: If $\frac{B+a-2 R-c}{d-c}<Q_{0} \quad$ or equivalently $R>\frac{1}{2}[B+$ $\left.a-c-Q_{0}(d-c)\right]$, then some customers buy the items from the list-price channel. Thus, the expected profit is

$$
\begin{aligned}
\Pi(R)= & \frac{B+a-2 R-c}{d-c}\left(\frac{\frac{B+a-c}{2}+R}{2}-w\right) \\
& +\lambda\left(1-\frac{B+a-2 R-c}{d-c}\right)(B-w)-C_{f} .
\end{aligned}
$$

We have, $\Pi(R)$ is concave in $R$. Solving the above equation yields $R^{*}=\hat{w}=w+\lambda(B-w)$.

The corresponding optimal profit is given by

$$
\begin{aligned}
\Pi^{*}(R)= & \frac{B+a-2(w+\lambda(B-w))-c}{d-c} \\
& \times\left(\frac{\frac{B+a-c}{2}+w+\lambda(B-w)}{2}-w\right) \\
& +\lambda\left(1-\frac{B+a-2(w+\lambda(B-w)-c}{d-c}\right) \\
& \times(B-w)-C_{f} .
\end{aligned}
$$

Case 2: If $Q_{0} \leq \frac{B+a-2 R-c}{d-c}$ or equivalently $R \leq \frac{1}{2}[B+a-$ $\left.c-Q_{0}(d-c)\right]$, then we obtain

$$
\begin{aligned}
\Pi(R)= & Q_{0}\left(\frac{\frac{B+a-c}{2}+R}{2}-w\right) \\
& +\lambda\left(1-Q_{0}\right)(B-w)-C_{f},
\end{aligned}
$$

which increases in $R$. So, we can infer that the optimal reserve price is obtain at the upper boundary value of $Q_{0} \leq \frac{B+a-2 R-c}{d-c}$, which is given by $R=\frac{1}{2}[B+a-c-$ $\left.Q_{0}(d-c)\right]$.

So, if $\hat{w}<\frac{1}{2}\left[B+a-c-Q_{0}(d-c)\right], R^{*}=\frac{1}{2}[B+a-c-$ $\left.Q_{0}(d-c)\right]$; otherwise, $R^{*}=\hat{w}$.

Proof of Proposition 4.1. In the double-bid scenario, the consumer's cost function is

$$
\begin{aligned}
\min _{x, \Delta} C(\theta)= & F(x)(x+\theta)+(1-F(x)) \\
& \times\left[F_{2}(x+\Delta)(x+\Delta+\theta)\right. \\
& \left.+\left(1-F_{2}(x+\Delta)\right) B\right] .
\end{aligned}
$$

Substituting $F(x)=\frac{x-a}{B-a^{\prime}} \quad f(x)=\frac{1}{B-a^{\prime}}, \quad F_{2}(x+\Delta)=\frac{\Delta}{B-x^{\prime}}$ and $f_{2}(x+\Delta)=\frac{\Delta}{(B-x)^{2}}$ into the above equation, we obtain

$$
\begin{aligned}
\min _{x, \Delta} C(\theta)= & \frac{x-a}{B-a}(x+\theta) \\
& +\frac{\Delta}{B-a}(x+\Delta+\theta)+\frac{B-x-\Delta}{B-a} .
\end{aligned}
$$


The second-order derivatives of $C(\theta)$ with respect to $x$ and $\Delta$ are given by

$$
\begin{aligned}
& \frac{\partial^{2} C(\theta)}{\partial x^{2}}=\frac{2}{B-a}>0, \\
& \frac{\partial^{2} C(\theta)}{\partial \Delta^{2}}=\frac{2}{B-a}>0, \\
& \frac{\partial^{2} C(\theta)}{\partial x \partial \Delta}=\frac{1}{B-a}>0 .
\end{aligned}
$$

Furthermore, Hessian matrix is

$$
\begin{aligned}
H & =\left|\begin{array}{ll}
\frac{\partial^{2} C(\theta)}{\partial x^{2}} & \frac{\partial^{2} C(\theta)}{\partial x \partial \Delta} \\
\frac{\partial^{2} C(\theta)}{\partial x \partial \Delta} & \frac{\partial^{2} C(\theta)}{\partial \Delta^{2}}
\end{array}\right|=\left|\begin{array}{cc}
\frac{2}{B-a} & \frac{1}{B-a} \\
\frac{1}{B-a} & \frac{2}{B-a}
\end{array}\right| \\
& =\frac{3}{(B-a)^{2}}>0 .
\end{aligned}
$$

Thus, $C(\theta)$ is strictly convex in $x$ and $\Delta$, and thus, the optimal solution of $x$ and $\Delta$ is unique.

Taking the first derivative of $C(\theta)$ with respect to $x$ and $\Delta$, respectively, yields

$$
\begin{aligned}
& \frac{\partial C(\theta)}{\partial x}=\frac{2 x+\theta+\Delta-a-B}{B-a}=0, \\
& \frac{\partial C(\theta)}{\partial \Delta}=\frac{2 \Delta+x+\theta-B}{B-a}=0 .
\end{aligned}
$$

Solving the above two equations results in the proposition.

Proof OF Proposition 4.2. We first start from abundant capacity cases. Owing to the similarity, we only consider the situation that $a<w<\frac{B+2 a-d}{3}$.

Case 1: Since $d \leq B+2 a-3 R$ and every customer wins with the first bid, $\Pi(R)=\left(\frac{\frac{B+2 a-c}{3}+R}{2}-w\right)$.

Case 2: $\quad c \leq B+2 a-3 R \leq d \leq B+\frac{1}{2} a-\frac{3}{2} R, \quad$ i.e., $R_{3}=\frac{B+2 a-d}{3} \leq R \leq \frac{2 B+a-2 d}{3}=R_{4}$, every customer wins with either the first bid or the second bid. The expected profit is

$$
\begin{aligned}
\Pi(R)= & \frac{B+2 a-3 R-c}{d-c}\left(\frac{\frac{B+2 a-c}{3}+R}{2}-w\right) \\
& +[1-P\{\theta \leq B+2 a-3 R\}]\left(\frac{3 R+B-a-d}{3}-w\right) \\
= & \frac{B+2 a-3 R-c}{d-c}\left(\frac{\frac{B+2 a-c}{3}+R}{2}-w\right) \\
& +\frac{d-(B+2 a-3 R)}{d-c}\left(\frac{3 R+B-a-d}{3}-w\right),
\end{aligned}
$$

where $\frac{3 R+B-a-d}{3}$ is the expected payoff of $x^{*}+\Delta^{*}=$ $\frac{2}{3} B+\frac{1}{3} a-\frac{2}{3} \theta$ on $B+2 a-3 R \leq \theta \leq d \leq B+\frac{1}{2} a-\frac{3}{2} R$.

Case 3: $R_{4}=\frac{2 B+a-2 d}{3} \leq R \leq \frac{B+2 a-c}{3}=R_{5}$, in which some customers' first bids are rejected but their second bids are accepted. In this scenario, some customers lose both bids. The expected profit is

$$
\begin{aligned}
& \Pi(R)=\frac{B+2 a-3 R-c}{d-c}\left(\frac{\frac{B+2 a-c}{3}+R}{2}-w\right) \\
& +\left[P\left\{\theta \leq B+\frac{1}{2} a-\frac{3}{2} R\right\}-P\{\theta \leq B+2 a-3 R\}\right] \\
& \left(\frac{3 R-a}{2}-w\right)=\frac{B+2 a-3 R-c}{d-c}\left(\frac{\frac{B+2 a-c}{3}+R}{2}-w\right) \\
& \quad+\frac{\frac{3}{2}(R-a)}{d-c}\left(\frac{3 R-a}{2}-w\right),
\end{aligned}
$$

where $\frac{3 R-a}{2}$ is the expected payoff of $x^{*}+\Delta^{*}=\frac{2}{3} B+$ $\frac{1}{3} a-\frac{2}{3} \theta$ on $B+2 a-3 R \leq \theta \leq B+\frac{1}{2} a-\frac{3}{2} R \leq d$.

Case 4: If $B+2 a-3 R \leq c \leq B+\frac{1}{2} a-\frac{3}{2} R$, then $R_{5}=\frac{B+2 a-c}{3} \leq R \leq \frac{2 B+a-2 c}{3}=R_{6}$, this implies that all customers' first bids are rejected while some of their second bids are accepted. We have

$$
\begin{aligned}
\Pi(R) & =P\left\{c \leq \theta \leq B+\frac{1}{2} a-\frac{3}{2} R\right\}\left(\frac{B+\frac{1}{2} a+\frac{3}{2} R-c}{3}-w\right) \\
& =\frac{B+\frac{1}{2} a-\frac{3}{2} R-c}{d-c}\left(\frac{B+\frac{1}{2} a+\frac{3}{2} R-c}{3}-w\right),
\end{aligned}
$$

where $\frac{B+\frac{1}{2} a+\frac{3}{2} R-c}{3}$ is the expected payoff of $x^{*}+\Delta^{*}=$ $\frac{2}{3} B+\frac{1}{3} a-\frac{2}{3} \theta$ on $c \leq \theta \leq B+\frac{1}{2} a-\frac{3}{2} R$.

Case 5: If $B+\frac{1}{2} a-\frac{3}{2} R<c$, then $R \geq \frac{2 B+a-2 c}{3}=R_{6}$. In this case no customer wins any item from the auction after bidding twice.

Integrating Cases 1-5 gives us a continuous curve.

In Case $1, \Pi(R)$ is increasing with respect to $R$ and the global optimal reserve price is given by the upper boundary $R_{3}$. Thus, the maximal profit is $\Pi(R)=\frac{2 B+4 a-c-d}{6}-w$.

In Case 2, $\Pi(R)$ is convex with the global minimum point $R=\frac{B+3 a-c-d}{4}$, which is basically smaller than the lower boundary $R_{3}$ given that $B-a>d$ and $c=0$ typically. Thus, $\Pi(R)$ increases when $R$ increases during $R_{3} \leq R \leq R_{4}$. A special case is that if $R_{3}=R_{4}$ then Case 2 disappears.

In Case 3, $\Pi(R)$ is convex with the global minimum point $R^{*}=2 a-w$. 
(1) If $2 a-w \leq R_{4}$, we can infer that $\Pi(R)$ increases with respect to $R$ during $R_{4} \leq R_{5}$ and $R^{*}=B$ $\frac{+2 a-c}{3}$.

(2) However, it is possible that $R_{4} \leq 2 a-w \leq R_{5}$, which is equivalent to $\frac{4 a-B+c}{3} \leq w \leq \frac{2 d+5 a-2 B}{3}$. Consider $\left[\frac{B+2 a-c}{3}-(2 a-w)\right]-\left[(2 a-w)-\frac{2 B+a-2 d}{3}\right]=$ $\frac{3 B-9 a+6 w-2 d-c}{3}$. Since $\Pi(R)$ is symmetric relative to $R^{*}=2 a-w$, if $\frac{4 a-B+c}{3} \leq w \leq \frac{2 d+9 a+c-3 B}{6}$ such that $\Pi(R)$ decreases in the domain of $\frac{2 B+a-2 d}{3} \leq 2 a-w \leq \frac{B+2 a-c}{3}$, the optimal reserve price is given by $R^{*}=\frac{2 B+a-2 d}{3}$.

(3) Otherwise if $\frac{2 d+9 a+c-3 B}{6} \leq w \leq \frac{2 d+5 a-2 B}{3}, \quad R^{*}=$ $\frac{B+2 a-c}{3}$ since overall $\Pi(R)$ increases in the same domain.

(4) If $R_{5} \leq 2 a-w$, i.e., $w \leq \frac{4 a-B+c}{3}, R^{*}=\frac{2 B+a-2 d}{3}$ since $\Pi(R)$ strictly decreases in the domain.

In Case $4, \Pi(R)$ is concave with the global maximum point $R^{*}=w$. If $w \leq \frac{B+2 a-c}{3}, \Pi(R)$ decreases between $R_{5} \leq R \leq R_{6}$ and thus $R^{*}=\frac{B+2 a-c}{3}$. If $w \geq R_{5}$, the optimal reserve price is given by $R^{*}=w$. It is worth noting that if $w \geq R_{6}, \Pi(R)=0$ since no bid can win the item from the NYOP auction.

Case 5 is trivial since no transaction occurs. Since the curve is continuously increasing in Cases 1 and 2, the optimal reserve price is basically determined in Cases 3 and 4. In summary:

(1) If $w \leq \frac{4 a-B+c}{3}, R^{*}=\frac{2 B+a-2 d}{3}$.

(2) If $\frac{4 a-B+c}{3} \leq w \leq \frac{2 d+9 a+c-3 B}{6}, R^{*}=\frac{2 B+a-2 d}{3}$.

(3) If $\frac{2 d+9 a+c-3 B}{6} \leq w \leq \frac{2 d+5 a-2 B}{3}, R^{*}=\frac{B+2 a-c}{3}$.

(4) If $\frac{2 d+5 a-2 B}{3} \leq w \leq \frac{B+2 a-c}{3}, R^{*}=\frac{B+2 a-c}{3}$.

(5) If $\frac{B+2 a-c}{3} \leq w \leq \frac{2 B+2 a-2 c}{3}, R^{*}=w$.

(6) If $w \geq \frac{B+2 a-c}{3}, R^{*}=w$.

The above conditions can be simplified into Equation (5). However, if $\frac{2 d+9 a+c-3 B}{6}<0$, Equation (5) can be rewritten as $R^{*}=\max \left\{\frac{B^{6}+2 a-c}{3}, w\right\}$.

Proof of Proposition 4.3. Taking into account the constrained capacity level $Q_{0}$ in the above discussion leads to the following cases. Owing to the lengthy computation, we only show the analysis when $\hat{Q}_{0}<\hat{Q}_{2}$ given that $B-a<d+(d-c) / 3$. The analysis on $\hat{Q}_{1} \geq \hat{Q}_{2}$ is similar and yields the same result.

Scenario B: $Q_{0}<\hat{Q}_{1}$

Case 1Q: In this scenario, Case 1Q overlaps with Cases 1 and 2, and part of Case 3. Since $Q_{0}<\hat{Q}_{1}$ cannot even satisfy the demand of the first bids, we have $\Pi(R)=Q_{0}\left(\frac{\frac{B+a-c}{2}+R}{2}-w\right)$.

Case 2Q: Case 2Q overlaps with parts of Cases 3 and 4. We consider two subcases here.
(1) $2 Q-1: R_{1} \leq R \leq R_{5}$ such that all first bids are satisfied and partial second bids are accepted as well (overlapping with Case 3). We have

$$
\begin{aligned}
\Pi(R)= & \frac{B+2 a-3 R-c}{d-c}\left(\frac{\frac{B+2 a-c}{3}+R}{2}-w\right) \\
& +\left(Q_{0}-\frac{B+2 a-3 R-c}{d-c}\right)\left(\frac{3 R-a}{2}-w\right) .
\end{aligned}
$$

(2) 2Q-2: $R_{51} \leq R \leq R_{2}$ such that all first bids are rejected and partial second bids are accepted (overlapping with Case 4). We have

$$
\Pi(R)=Q_{0}\left(\frac{B+\frac{1}{2} a+\frac{3}{2} R-c}{3}-w\right) .
$$

Case 3Q: $R>R_{2}$ such that all first bids are rejected and all second bids are accepted as it is in Case 4 .

$$
\Pi(R)=\frac{B+\frac{1}{2} a-\frac{3}{2} R-c}{d-c}\left(\frac{B+\frac{1}{2} a+\frac{3}{2} R-c}{3}-w\right) .
$$

A continuous curve of Scenario B is formed by Cases 1Q-3Q. In Case 1Q, $\Pi(R)$ is strictly increasing in $R$. Case 2Q-1 has a convex $\Pi(R)$ with respect to $R$. The global minimum point is given by $R^{*}=\frac{B+3 a-c-Q_{0}(d-c)}{4}$. Compare $R^{*}=\frac{B+3 a-c-Q_{0}(d-c)}{4}$ to $\frac{B+2 a-c-Q_{0}(d-c)}{3}$, the lower bound. Since $Q_{0}<\hat{Q}_{1}=\frac{B-a-c}{2(d-c)^{\prime}}$, we find that $R^{*}=$ $\frac{B+3 a-c-Q_{0}(d-c)}{4}$ is smaller than $R_{1}=\frac{B+2 a-c-Q_{0}(d-c)}{3}$ as shown as follows:

$$
\begin{aligned}
& \frac{B+2 a-c-Q_{0}(d-c)}{3}-\frac{B+3 a-c-Q_{0}(d-c)}{4} \\
& =\frac{B-a-c-Q_{0}(d-c)}{12} \geq 0 .
\end{aligned}
$$

Thus, Case $2 \mathrm{Q}-1$ is increasing in $R$. Since $\Pi(R)$ is increasing in Case 2Q-2, $\Pi(R)$ increases in Case 2Q. Case $3 \mathrm{Q}$ is part of Case 4 in the abundant capacity case. Thus, the abundant capacity curve and this constrained capacity curve intercept at $R=R_{2}=$ $\frac{2 B+a-2 c-2 Q_{0}(d-c)}{3}$. From our discussion in the proof of Proposition 4.2, we can infer that the optimal reserve price is given by $R_{2}$ if $R_{2}>w$; otherwise, it is given by $w$. Overall, $R^{*}=\max \left\{R_{2}, w\right\}$.

Scenario C: $\hat{Q}_{1} \leq Q_{0} \leq \hat{Q}_{2}$

Case 1Q: This is the same as in Scenario B: $\Pi(R)=Q_{0}\left(\frac{\frac{B+a-c}{2}+R}{2}-w\right)$.

Case 2Q: Case 2Q overlaps with part of Case 3 only. Thus, $R_{1} \leq R \leq R_{2}$ such that all first bids are satisfied 
and partial second bids are accepted as well. We have

$$
\begin{aligned}
\Pi(R)= & \frac{B+2 a-3 R-c}{d-c}\left(\frac{\frac{B+2 a-c}{3}+R}{2}-w\right) \\
& +\left(Q_{0}-\frac{B+2 a-3 R-c}{d-c}\right)\left(\frac{3 R-a}{2}-w\right) .
\end{aligned}
$$

Case 3Q: There are two subcases.

(1) $3 Q-1: R_{2} \leq R \leq R_{5}$ such that all first bids and second bids are accepted as it is in Case 3. We have

$$
\begin{aligned}
\Pi(R)= & \frac{B+2 a-3 R-c}{d-c}\left(\frac{\frac{B+2 a-c}{3}+R}{2}-w\right) \\
& +\frac{\frac{3}{2}(R-a)}{d-c}\left(\frac{3 R-a}{2}-w\right) .
\end{aligned}
$$

(2) $3 Q-2: R \geq R_{5}$ such that all first bids are rejected and all second bids are accepted as Case 4 . We have

$$
\Pi(R)=\frac{B+\frac{1}{2} a-\frac{3}{2} R-c}{d-c}\left(\frac{B+\frac{1}{2} a+\frac{3}{2} R-c}{3}-w\right) .
$$

Similarly, a curve of Cases 1Q-3Q crosses the curve of Cases 1-4. But, the interception point is located in Case 3. If $R^{*}=R_{2}$, it must have $\left[(2 a-w)-R_{2}\right]-$ $\left[R_{5}-(2 a-w)\right]>0$, which is equivalent to

$$
w<\frac{9 a-3 B+c+2 d-2\left(1-Q_{0}\right)(d-c)}{6}<\frac{9 a-3 B+c+2 d}{6} ;
$$

otherwise $R^{*}=R_{5}$. Overall, the optimal reserve price can be written as follows: (overlapping with Case 2). We have

$$
\begin{aligned}
\Pi(R)= & \frac{B+2 a-3 R-c}{d-c}\left(\frac{\frac{B+2 a-c}{3}+R}{2}-w\right) \\
& +\left(Q_{0}-\frac{B+2 a-3 R-c}{d-c}\right) \\
& \times\left(\frac{3 R+B-a-d}{3}-w\right) .
\end{aligned}
$$

(2) 2Q-2: $R_{4} \leq R \leq R_{2}$ such that all first bids are satisfied and partial second bids are accepted (overlapping with Case 3). We have

$$
\begin{aligned}
\Pi(R)= & \frac{B+2 a-3 R-c}{d-c}\left(\frac{\frac{B+2 a-c}{3}+R}{2}-w\right) \\
& +\left(Q_{0}-\frac{B+2 a-3 R-c}{d-c}\right)\left(\frac{3 R-a}{2}-w\right) .
\end{aligned}
$$

Case 3Q: There are two subcases.

(1) 3Q-1: $R_{2} \leq R \leq R_{5}$ such that all first bids and some second bids are accepted as Case 3 . We have

$$
\begin{aligned}
\Pi(R)= & \frac{B+2 a-3 R-c}{d-c}\left(\frac{\frac{B+2 a-c}{3}+R}{2}-w\right) \\
& +\frac{\frac{3}{2}(R-a)}{d-c}\left(\frac{3 R-a}{2}-w\right) .
\end{aligned}
$$

(2) $3 Q-2: R \geq R_{5}$ such that all first bids are rejected and all second bids are accepted as it is in

$$
\begin{cases}\text { If } w<\frac{9 a-3 B+c+2 d-2\left(1-Q_{0}\right)(d-c)}{6}, & R^{*}=\frac{2 B+a-2 c-2 Q_{0}(d-c)}{3}, \\ \text { if } \frac{9 a-3 B+c+2 d-2\left(1-Q_{0}\right)(d-c)}{6} \leq w \leq \frac{B+2 a-c}{3}, & R^{*}=\frac{B+2 a-c}{3}, \\ \text { if } w>\frac{B+2 a-c}{3}, & R^{*}=w .\end{cases}
$$

\section{Scenario $D: Q_{0}>\hat{Q}_{2}$}

Case 1Q: Case 1Q overlaps with Case 1 and part of Case 2. The profit function is the same $\Pi(R)=Q_{0}$ $\left(\frac{\frac{B+a-c}{2}+R}{2}-w\right)$ as long as $R \geq R_{1}$.

Case 2Q: Case 2Q overlaps with Case 2 and part of Case 3.

(1) 2Q-1: $R_{1} \leq R \leq R_{4}$ such that all first bids are satisfied and partial second bids are accepted
Case 4. We have

$$
\Pi(R)=\frac{B+\frac{1}{2} a-\frac{3}{2} R-c}{d-c}\left(\frac{B+\frac{1}{2} a+\frac{3}{2} R-c}{3}-w\right) .
$$

In Scenario $\mathrm{D}$, we directly consider subcase 2Q-1. The optimal reserve price in this sub-domain is given by $R=\frac{3 a+d-c-Q_{0}(d-c)}{3}$, which is smaller than $R_{1}$ due to $B-a>d$. Therefore, $\Pi$ increases in 2Q-1 with 
respect to $R$. Other parts of the curves share similar features with Scenario $C$ and the interception point is located at Case 3 as well. Overall, the optimal reserve price can be written as follows:
Case 3: $R_{4} \leq R \leq R_{5}$, unsatisfied customers start to purchase from the retailer-own list-price channel. The expected profit is

$$
\begin{cases}\text { If } w<\frac{9 a-3 B+c+2 d-2\left(1-Q_{0}\right)(d-c)}{6}, & R^{*}=\frac{2 B+a-2 c-2 Q_{0}(d-c)}{3}, \\ \text { if } \frac{9 a-3 B+c+2 d-2\left(1-Q_{0}\right)(d-c)}{6} \leq w \leq \frac{B+2 a-c}{3}, & R^{*}=\frac{B+2 a-c}{3}, \\ \text { if } w>\frac{B+2 a-c}{3}, & R^{*}=w .\end{cases}
$$

The difference between Scenarios $C$ and D lies at that $\frac{9 a-3 B+c+2 d-2\left(1-Q_{0}\right)(d-c)}{6}$ is a higher value in Scenario $\mathrm{D}$, which means that it is more likely that $R_{2}$ could be the optimal reserve price.

In summary, we can integrate the above scenarios into the following:

(1) If $Q_{0}<\hat{Q}_{1}$,

$$
R^{*}=\max \left\{\frac{2 B+a-2 c-2 Q_{0}(d-c)}{3}, w\right\}
$$

(2) Otherwise if $Q_{0} \geq \hat{Q}_{1}$, then

$$
\left\{\begin{array}{l}
\text { if } w<\frac{9 a-3 B+c+2 d-2\left(1-Q_{0}\right)(d-c)}{6} \\
\text { if } \frac{9 a-3 B+c+2 d-2\left(1-Q_{0}\right)(d-c)}{6} \leq w \leq \\
\text { if } w>\frac{B+2 a-c}{3}
\end{array}\right.
$$

Proof of Proposition 4.5. Owing to our special focus, the analysis of dual-channel scenario is similar to that of the single-channel scenario. Again, because of the lengthy computation, we only show the analysis when $\hat{Q}_{1}<\hat{Q}_{2}$. The analysis on $\hat{Q}_{1} \geq \hat{Q}_{2}$ is similar and yields the same result.

Case 1: $R \leq R_{3}$. Since no customer switches to the listprice channel, the expected profit is $\Pi(R)=$ $\left(\frac{\frac{B+2 a-c}{3}+R}{2}-w\right)-C_{f}$.

Case 2: $R_{3} \leq R \leq R_{4}$. Since all bidders win with either the first bid or the second bid, the expected profit is

$$
\begin{aligned}
\Pi(R)= & \frac{B+2 a-3 R-c}{d-c}\left(\frac{\frac{B+2 a-c}{3}+R}{2}-w\right) \\
& +\frac{d-(B+2 a-3 R)}{d-c}\left(\frac{3 R+B-a-d}{3}-w\right)-C_{f} .
\end{aligned}
$$

$$
\begin{aligned}
\Pi(R)= & \frac{B+2 a-3 R-c}{d-c}\left(\frac{\frac{B+2 a-c}{3}+R}{2}-w\right) \\
& +\frac{\frac{3}{2}(R-a)}{d-c}\left(\frac{3 R-a}{2}-w\right) \\
& +\lambda\left(1-\frac{B+2 a-3 R-c}{d-c}-\frac{\frac{3}{2}(R-a)}{d-c}\right) \\
& (B-w)-C_{f} .
\end{aligned}
$$

Case 4: $R_{5} \leq R \leq R_{6}$. The expected profit is

$$
\begin{aligned}
\Pi(R)= & \frac{B+\frac{1}{2} a-\frac{3}{2} R-c}{d-c}\left(\frac{B+\frac{1}{2} a+\frac{3}{2} R-c}{3}-w\right) \\
& +\lambda\left(1-\frac{B+\frac{1}{2} a-\frac{3}{2} R-c}{d-c}\right)(B-w)-C_{f}
\end{aligned}
$$

Case 5: If $R \geq R_{6}$, no customer wins any item from the NYOP auction after bidding twice. However, some customers buy from the list-price channel. $\Pi(R)=$ $\lambda(B-w)-C_{f}$.

Integrating Cases 1-5 gives us a continuous curve. Cases 1 and 2 behave similarly as in the single-channel situation as described in the proof of Proposition 4.2. For Case 3, $\Pi(R)$ is convex with the global minimum point $R^{*}=2 a-w-\lambda(B-w)$. Compared with $R^{*}=2 a-w$ in the single-channel case, $R^{*}=2 a-(w+$ 
$\lambda(B-w))$ is a smaller value. In Case $4, \Pi(R)$ is concave with the global maximum point $R^{*}=w+\lambda(B-w)$. Thus, we define a new variable $\hat{w}=w+\lambda(B-w)$. Similar to Proposition 4.2, the optimal reserve price is given by

$$
\begin{cases}\text { If } \hat{w}<\frac{2 d+9 a+c-3 B}{6}, & R^{*}=\frac{2 B+a-2 d}{3} \\ \text { if } \frac{2 d+9 a+c-3 B}{6} \leq \hat{w} \leq \frac{B+2 a-c}{3}, & R^{*}=\frac{B+2 a-c}{3} \\ \text { if } \hat{w}>\frac{B+2 a-c}{3}, & R^{*}=\hat{w} .\end{cases}
$$

The analysis on the optimal reserve price in Cases 1Q-3Q of the dual-channel scenario is similar to that in the single-channel scenario. Thus, we can integrate the above scenarios as follows:

(1) If $Q_{0}<\hat{Q}_{1}$,

$$
R^{*}=\max \left\{\frac{2 B+a-2 c-2 Q_{0}(d-c)}{3}, \hat{w}\right\}
$$

(2) Otherwise if $Q_{0} \geq \hat{Q}_{1}$, then

$$
\begin{cases}\text { if } \hat{w}<\frac{9 a-3 B+c+2 d-2\left(1-Q_{0}\right)(d-c)}{6}, & R^{*}=\frac{2 B+a-2 c-2 Q_{0}(d-c)}{3}, \\ \text { if } \frac{9 a-3 B+c+2 d-2\left(1-Q_{0}\right)(d-c)}{6} \leq \hat{w} \leq \frac{B+2 a-c}{3}, & R^{*}=\frac{B+2 a-c}{3} \\ \text { if } \hat{w}>\frac{B+2 a-c}{3}, & R^{*}=\hat{w} .\end{cases}
$$

In Cases 1Q-3Q, due to the similarity, we just demonstrate $2 \mathrm{Q}$ in Scenario $\mathrm{B}$.

Case 2Q: Case 2Q overlaps with parts of Cases 3 and 4. We consider two subcases here.

(1) $2 Q-1: R_{1} \leq R \leq R_{5}$ such that all first bids are satisfied and partial second bids are accepted (overlapping with Case 3). We have

$$
\begin{aligned}
\Pi(R)= & \frac{B+2 a-3 R-c}{d-c}\left(\frac{\frac{B+2 a-c}{3}+R}{2}-w\right) \\
& +\left(Q_{0}-\frac{B+2 a-3 R-c}{d-c}\right)\left(\frac{3 R-a}{2}-w\right) \\
& +\lambda\left(1-Q_{0}\right)(B-w)-C_{f} .
\end{aligned}
$$

(2) $2 Q-2: R_{5} \leq R \leq R_{2}$ such that all first bids are rejected and partial second bids are accepted (overlapping with Case 4). We have

$$
\begin{aligned}
\Pi(R)= & Q_{0}\left(\frac{B+\frac{1}{2} a+\frac{3}{2} R-c}{3}-w\right) \\
& +\lambda\left(1-Q_{0}\right)(B-w)-C_{f} .
\end{aligned}
$$

\section{References}

Allbusiness.com (1999). Online grocers see new twist with priceline.com's entrance. Food E Drink Weekly. September 27, 1999 issue.

Cai, G., P. Wurman. (2005). Monte Carlo approximation in incomplete-information, sequential-auction games. Decision Support Syst. 39(2): 153-168.

Cai, G., Z. G. Zhang, M. Zhang. (2009). Game theoretical perspectives on dual-channel supply chain competition with price discounts and pricing schemes. Int. J. Prod. Econ., 117(1): 80-96.

Caldentey, R., G. Vulcano. (2007). Online auction and list price revenue management. Manage. Sci. 53(5): 795-813.

Chen, F. Y., J. Chen, Y. Xiao. (2007). Optimal control of selling channels for an online retailer with cost-per-click payments and seasonal products. Prod. Oper. Manag. 16(3): 292-305.

Chen, Y.-J., S. Seshadri, E. Zemel. (2008). Sourcing through auctions and audits. Prod. Oper. Manag. 17(2): 121-138.

Chernev, A. (2003). Name-your-own-price online: Price elicitation strategies in consumer choice. J. Consum. Psychol. 13(1/2): 51-62.

Chiang, W. K., D. Chhajed, J. D. Hess. (2003). Direct marketing, indirect profits: A strategic analysis of dual-channel supply chain design. Manage. Sci. 49(1): 1-20.

Clark, B. (2000). We put groceries at Priceline to the test. Money 29(3): 167-170.

Ding, M., J. Eliashberg, J. Huber, R. Saini. (2005). Emotional bidders an analytical and experimental examination of consumers behavior in priceline-like reverse auctions. Manage. Sci. 51(3): 352364.

Etzion, H., E. Pinker, A. Seidmann. (2006). Analyzing the simultaneous use of auctions and posted prices for on-line selling. Manuf. Serv. Oper. Manage. 8: 68-91.

Fay, S. (2004). Partial repeat bidding in the name-your-own-price channel. Mark. Sci. 23(3): 407-418.

Hann, I.-H., C. Terwiesch. (2003). Measuring the frictional cost of online transactions: The case of a name-your-own-price channel. Manage. Sci. 49(11): 1563-1579. 
Rothkopf, M. H., A. B. Whinston. (2007). On e-auctions for procurement operations. Prod. Oper. Manag. 16(4): 404-408.

Segan, S. (2005). Priceline.com for Dummies. Wiley Publishing, New York.

Shen, Z.-J. M., X. Su. (2007). Customer behavior modeling in revenue management and auctions: A review and new research opportunities. Prod. Oper. Manag. 16(6): 713-728.

Spann, M., M. Bernhardt, G. Häubl, B. Skiera. (2005). It's all in how you ask: Effects of price-elicitation format on bidding behavior in reverse pricing markets. In Proceedings of the 26th Annual Conference of the Society for Judgment and Decision Making (SJDM) Toronto, Canada.

Spann, M., B. Skiera, B. Schfers. (2004). Measuring individual frictional costs and willingness-to-pay via name-your-own-price mechanisms. J. Interact. Mark. 18(4): 22-36.

Spann, M., G. J. Tellis. (2006). Does the internet promote better consumer decisions? The case of name-your-own-price auctions. J. Mark. 70(1): 65-78.
Terwiesch, C., S. Savin, I.-H. Hann. (2005). Online-haggling at a name-your-own-price retailer: Theory and application. Manage. Sci. 51(3): 339-351.

Tsay, A. A., N. Agrawal. (2004). Channel conflict and coordination in the e-commerce age. Prod. Oper. Manag. 13(1): 93-110.

van Ryzin, G., G. Vulcano. (2004). Optimal auctioning and ordering in an infinite horizon inventory pricing system. Oper. Res. 52: 346-367.

Wang, T., E. Gal-Or, R. Chatterjee. (2005). Why a 'name-your-ownprice' channel makes sense for service providers. Working paper, University of Pittsburgh.

Wilson, J., G. Zhang. (2008). Optimal design of a name-your-ownprice channel when customers behave strategically. J. Revenue Pricing Manage. 7(3): 281-290.

Zhao, X. (2008). Coordinating a supply chain system with retailers under both price and inventory competition. Prod. Oper. Manag. 17(5): 532-542. 